\title{
Structural distortion, charge modulation and local anisotropies in magnetite below the Verwey transition using resonant $x$-ray scattering
}

\author{
Gloria Subías, ${ }^{\text {a }}$ Joaquín García, ${ }^{\text {a Javier Blasco, }{ }^{a} \text { Javier Herrero-Martín, }}$ \\ M. Concepción Sánchez, ${ }^{a}$ Julia Orna ${ }^{\text {ac }}$ and Luis Morellón ${ }^{\text {ac }}$
}

alnstituto de Ciencia de Materiales de Aragón, CSIC-Universidad de Zaragoza, Departamento de Física de la Materia Condensada, Pedro Cerbuna 12, 50009, Zaragoza, Spain, ${ }^{b}$ Institut de Ciència de Materials de Barcelona-CSIC, Campus Universitari de Bellaterra, 08193 Bellaterra, Spain, and 'Instituto de Nanociencia de Aragón, Departamento de Física de la Materia Condensada, Universidad de Zaragoza, 50009 Zaragoza, Spain. E-mail: gloria@unizar.es

Synopsis The complete description of the structural changes, charge disproportions and local anisotropies below the Verwey transition in magnetite is provided by means of resonant $\mathrm{x}$-ray scattering data in high-quality single crystals and thin films grown on $\mathrm{MgO}$.

\begin{abstract}
The pattern of charge modulations and local anisotropies below the Verwey transition has been determined and quantified in high quality $\mathrm{Fe}_{3} \mathrm{O}_{4}$ single crystals and thin films grown on MgO by using resonant x-ray scattering at the Fe K-edge. The energy, polarization and azimuthal angle dependencies of an extensive set of reflections with potential sensitivity to charge or local anisotropy orderings have been analyzed to explore their origins. A charge disproportion on octahedral B sites of $0.20 \pm 0.05 \mathrm{e}^{-}$with [ $\left[\begin{array}{lll}0 & 0 & 1\end{array}\right]$ and [ $\left[\begin{array}{lll}1 & -1 & 0\end{array}\right]$ cubic periodicities has been confirmed, while no charge disproportion has been obtained with [0 0 $1 / 2$ ] cubic periodicity. Additional charge modulations in the monoclinic $a-b$ plane are also present. In addition, the occurrence of new forbidden $(1,1,0)$ and $(0,0,2 n+1 / 2)$ cubic reflections that arise from the anisotropy of the local structure around different tetrahedral and octahedral Fe atoms is shown. This complex pattern of weak charge modulations and local anisotropies is fully compatible with the low temperature crystal structure refined in the nonpolar $C 2 / c$ space group and disprove any bimodal charge disproportion of the octahedral Fe atoms.
\end{abstract}

Keywords: Resonant x-ray scattering; Verwey transition; charge and orbital order; mixed valence oxides 


\section{Introduction}

Magnetite $\left(\mathrm{Fe}_{3} \mathrm{O}_{4}\right)$ undergoes a first order metal-insulator and structural transition at $\mathrm{T}_{\mathrm{V}}$ 124 K (Verwey transition (Verwey, 1939; Verwey \& Haayman, 1941) that has been considered for a long time as the example of a charge-ordering transition (CO), in which a simple ionic mechanism determines the electronic properties (Anderson, 1956). Despite the Verwey model was disproved by experiments 30 years ago (García \& Subías, 2004), the idea of a periodic ordering of localized electrons has survived until recently (Imada et al., 1998).

The crystallographic unit cell at room temperature is the inverse spinel cubic cell, with space group $F d-3 m$ (indicated by the subscript $\mathrm{C}$ in the text). The iron atom is located in sites with tetrahedral A-site and octahedral B-site coordination. To reflect the mixed valence nature of magnetite, the chemical formula is sometimes written as $\mathrm{Fe}_{A}{ }^{3+}\left[\mathrm{Fe}_{B}{ }^{3+}, \mathrm{Fe}_{B}{ }^{2+}\right] \mathrm{O}_{4}$. Verwey (Verwey \& Haayman, 1941) proposed that the discontinuity in the electrical resistivity is caused by the ionic ordering of $\mathrm{Fe}^{2+}$ and $\mathrm{Fe}^{3+}$ at the $\mathrm{B}$-sites in planes perpendicular to the $c$ axis, giving rise to a superstructure of ordered charges in agreement with Anderson criteria (Anderson, 1956) for CO. The structural refinement made by Iizumi et al. (Iizumi et al., 1982) on a partially detwinned single crystal by neutron diffraction revealed a crystal distortion incompatible with the Verwey model. They found that the crystallographic symmetry would be monoclinic in the $C c$ space group which implies a total of 16 nonequivalent B-sites but a reasonable approximation to the real structure was obtained using the Pmca constraints for the atomic positions, which yields only four independent B1, B2, B3 and B4 sites. Indeed, no significant variation in mean Fe-O distances for the octahedral sites was revealed, such might be expected to accompany $\mathrm{CO}$. More recently,_neither $\mathrm{Fe}^{3+}$ nor $\mathrm{Fe}^{2+}$ ions have been identified at the octahedral B sites by nuclear magnetic resonance (Novák et al., 2000) and resonant x-ray scattering (RXS) (Kanazawa et al., 2002; García et al., 2000; García et al., 2001) works, and, if any kind of CO occurs, the difference in charge between distinct crystallographic sites was found to be lower than 0.25 electrons. However, despite the small value of the charge disproportion $(\delta)$ on the B sub-lattice, the presence of $\mathrm{CO}$ in magnetite is still being debated from experimental studies (Wright et al., 2001; Wright et al., 2002; Subías et al., 2004a; Nazarenko et al., 2006; Joly et al., 2008; García et al., 2009) and theoretical calculations (Szotek et al., 2003; Leonov et al., 2004; Jeng et al., 2004; Madsen \& Novák, 2005; Rowan et al., 2009).

By combining high-resolution neutron and x-ray powder diffraction data, Wright et al. (Wright et al., 2001; Wright et al., 2002) have obtained a P2/C monoclinic phase for the low temperature structure. $P 2 / C$ and Pmca cells are metrically equivalent but in $P 2 / C$ there are six non-equivalent octahedral sites (B1a, B1b, B2a, B2b, B3 and B4). However, the refinement was only stable under the Pmca constraints and divided the B-sites into two groups based on a 
bond valence sum (BVS) analysis (Brese \& O’Keeffe, 1991). B1 (B1a+B1b) and B4 sites have an average valence of +2.4 whereas B2 $(B 2 a+B 2 b)$ and B3 sites have an average valence of +2.6 . Despite this small charge segregation, the bimodal charge distribution was still interpreted in terms of a $\mathrm{Fe}^{2+}-\mathrm{Fe}^{3+}$ ionic ordering (Wright et al., 2001; Wright et al., 2002). Recently, a symmetry mode analysis has allowed the refinement of magnetite in the C2/c monoclinic cell, which is metrically equivalent to the Cc cell (Blasco et al., 2011). In this C2/c model, each B1b, B2a, B3 and B4 sites are split into two non-equivalent sites, resulting in a total of 10 independent octahedral sites with a multimodal distribution of valences ranging between 2.53 and 2.84, as estimated from BVS. Hereafter we will refer to the three relevant structural descriptions as Pmca-, P2/c- and $C 2 / c$-models. The arrangement of Fe ions in A and B sites is illustrated in figure 1 for the $C 2 / c$-model. The same notation as in reference (Blasco et al., 2011) is used.

So far, most theoretical calculations have been done using the P2/c-model (Wright et al., 2001; Wright et al., 2002) and, accordingly, they found small charge disproportions of the octahedral sites in fair agreement with Wright's refinement. However, they still proposed that conduction electrons are fully localized on the electron-rich $\mathrm{Fe}^{2+} \mathrm{B} 1 / \mathrm{B} 4$ sites resulting in an orbital ordering (OO) of the occupied $\mathrm{t}_{2 \mathrm{~g}}$ states (Leonov et al., 2004; Jeng et al., 2004; Madsen \& Novák, 2005; Rowan et al., 2009). Only Szotek et al. (Szotek et al., 2003) proposed that structural distortions, rather than localization/delocalization correlations, are responsible for the charge disproportion in the low-temperature phase. This coupling between structural and electronic mechanisms for the Verwey transition has been recently supported by new theoretical calculations (Pinto \& Elliot, 2006; Piekarz et al., 2007).

RXS is the ideal technique to solve questions about the presence and magnitude of periodic arrangements of charge and/or local anisotropies (Subías et al., 2009). The physical reason is the strong contrast in the atomic anomalous scattering factor at energies close to the absorption edge, either between ions with different valence states, or between equivalent atoms with a differently oriented local anisotropy. The atomic scattering factor for an atom is usually written as $f=f^{0}+f^{\prime}(E)+$ if $f^{\prime \prime}(E)$. This contains an energy independent part, $f^{0}$, corresponding to the classical Thomson scattering, and two energy-dependent parts, $f^{\prime}(E)+$ if $"(E)$ known as the anomalous terms. For electric dipole-dipole transitions, the atomic anomalous scattering factor is a tensor of the polarization vectors that can be expressed as

$$
f^{\prime}(E)+i f^{\prime \prime}(E)=\sum_{m, n} \varepsilon_{m} \varepsilon_{n}^{\prime} \hat{S}_{m n}(E)
$$


where $\varepsilon$ and $\varepsilon$ represent the incident and scattered photon polarization, respectively, and $\hat{S}_{m n}$ is a symmetric tensor of second rank where the sum runs over the Cartesian coordinates $\mathrm{x}, \mathrm{y}, \mathrm{z}$. A common approach is to consider only the isotropic part of this tensor, i.e. the anomalous term is thus scalar. This is appropriate when the main contrast between the scattered atoms comes from a different charge density on different crystallographic sites (charge disproportion). On the other hand, the tensor character of the anomalous term is manifested in the observation of resonant intensity in forbidden reflections. These reflections are still allowed even if the scalar parts becomes zero due to the systematic absences of (nonsymmorphic) symmetry operations and are referred to as ATS (anisotropy of the tensor of susceptibility) reflections (Dmitrienko et al., 2005). Initial RXS studies have reported the presence of forbidden $(0, k, l)_{\mathrm{C}}$ with $k+l=4 \mathrm{n}+2$ reflections on magnetite above the Verwey transition (Kanazawa et al., 2002; García et al., 2000). These studies showed that those forbidden reflections were not related to $\mathrm{CO}$, but they resulted from an anisotropic ordering of the trigonal distortion on the octahedral Fe atoms. This RXS signal does not change by cooling below $\mathrm{T}_{\mathrm{V}}$, and therefore, a limit of $0.25 \mathrm{e}^{-}$was established for any possible charge disproportion between the octahedral sites (García et al., 2001). Later, RXS experiments on reflections following the $\left[\begin{array}{lll}0 & 0 & l\end{array}\right]_{\mathrm{C}}$ periodicity showed that the charge disproportion, if present, would be very small (Subías et al., 2004a). Another recent RXS experiment (Nazarenko et al., 2006) was analyzed based on the $P 2 / c$-model. They found a $\left[\begin{array}{lll}0 & 0 & 1\end{array}\right]_{\mathrm{C}}$ charge modulation of about 0.2e- between the B1 and B2 iron atoms along the cubic $c$-axis in overall agreement with the CO proposed by the P2/C-model. A similar charge disproportion between B3 and B4 sites with a $\left[\begin{array}{lll}0 & 0 & 1 / 2\end{array}\right]_{\mathrm{C}}$ periodicity was also proposed, but this study was inconclusive since the studied resonant reflections are not sensitive to the charge difference between these two sites (García et al., 2007). Indeed, the same authors imposed no disproportion between B3 and B4 atoms in their following work (Joly et al., 2008). In addition, Goff et al. (Goff et al., 2005) deduced a significant charge disproportion (46\%) between B1 and B2 sites in the full CC monoclinic structure after the refinement of powder resonant x-ray diffraction patterns. Although the $\left[\begin{array}{lll}0 & 0 & 1\end{array}\right]_{\mathrm{C}}$-type charge modulation between B1 and B2 sites has been confirmed (García et al., 2009), the existence of a charge disproportion between B3 and B4 sites and, consequently, the proposed bimodal $\mathrm{Fe}^{+(2.5+\delta)}-\mathrm{Fe}^{+(2.5-\delta)}$ distribution, still remains uncertain. Moreover, such a bimodal pattern does not agree with the more complete $C 2 / c$ model.

This controversy also exists in the interpretation of the soft RXS studies at the O-K (Huang et al., 2006; Wilkins et al., 2009) and Fe $\mathrm{L}_{2,3}$-edges (Schlappa et al., 2008) of the (0, $0, l / 2)_{\mathrm{C}}$ type reflections. The observed resonant scattering at the $(0,0,1 / 2)_{\mathrm{C}}$ reflection was considered as a direct evidence for a $\mathrm{Fe}^{3+} / \mathrm{Fe}^{2+} \mathrm{CO}-\mathrm{OO}$ (Huang et al., 2006; Schlappa et al., 2008). This interpretation has been further supported by Lorenzo et al. (Lorenzo et al., 2008), 
who also considered the observation of a resonance at the Fe K-edge in this half-integer reflection as a signature of OO. However, the most recent RXS works at the Fe K-edge explained these as ATS reflections due to the existence of the c glide-plane symmetry without requiring any OO (García et al., 2009; Bland et al., 2009). This was the same conclusion reached by Wilkins et al. (Wilkins et al., 2009) when reanalyzing the O K-edge RXS data.

The goal of the present work is to provide a consistent description of the structural changes, charge disproportions and local anisotropies present in the low temperature phase of magnetite. Our strategy was the following: i) we have performed RXS measurements at the Fe K-edge on two types of samples, highly stoichiometric single-crystals and thin films ( $t \sim 200 \mathrm{~nm}$ ) grown on MgO substrates. The latter provide us data with negligible selfabsorption directly from the experiment. We note that a proper correction for the strong selfabsorption that affects the RXS data for single-crystals is one of the major difficulties for a reliable quantitative analysis of tiny charge modulations. Moreover, the comparison between two independent sets of data guarantees us the consistency of our results. Polarization and azimuthal angle dependencies of an extensive set of reflections with potential sensitivity to either charge or local anisotropy orderings were studied. ii) The origin of the different resonant reflections (charge or anisotropy) is discussed in terms of the structural changes at the Verwey transition. A quantitative analysis of the possible charge disproportions was carried out based on the $P 2 / C$ and $C 2 / C$ structural models. The key feature of our work is that simulations and fit of the RXS intensities have been carried out solely based on experimental data. iii) To conclude, we compare these new results with previous RXS studies and propose a complete description of the changes in the structural and electronic properties of magnetite at the Verwey transition that supports an itinerant electronic model.

\section{Experimental}

Magnetite was synthetically prepared by using high purity $\mathrm{Fe}_{2} \mathrm{O}_{3}(99.999 \%)$ that was fired at $1200{ }^{\circ} \mathrm{C}$ for $24 \mathrm{~h}$ in a current flow of $\mathrm{CO}_{2} / \mathrm{CO}(96 / 4)$. The powders were ground, pressed into rods, and sintered at $1400^{\circ} \mathrm{C}$ for $24 \mathrm{~h}$ in the same atmosphere, which ensures the right cation to oxygen stoichiometry (Aragón et al., 1984). The single crystals were grown from the rods in the same atmosphere by the floating zone method (Blasco et al., 2008b). X-ray powder diffraction of the crushed crystals guaranteed the phase purity. The temperature dependence of the initial ac magnetic susceptibility showed a sharp discontinuity at $T_{V}=123.5$ $\mathrm{K}$ with a narrow transition width, $\Delta \mathrm{T}=1 \mathrm{~K}$, and the saturation magnetization was $4.11 \mu_{\mathrm{B}}$ at 5 K (García et al., 2009). These features indicate that the samples were stoichiometrically correct within the first order transition region [34; Shepherd et al., 1991). Two crystals, labeled as $\mathrm{A}$ and $\mathrm{B}$, were cut with the $[001]_{\mathrm{C}}$ and the $[110]_{\mathrm{C}}$ directions along the surface normal, respectively. High quality thin films also labeled as A and B of thickness about 250 
$\mathrm{nm}$ were grown on $<100>\mathrm{MgO}$ and $<110>\mathrm{MgO}$ substrates, respectively by pulsed laser deposition using a $\mathrm{KrF}$ excimer laser with $248 \mathrm{~nm}$ wavelength and $10 \mathrm{~Hz}$ repetition rate in an ultrahigh-vacuum chamber (De Teresa et al., 2007). The close lattice match between MgO and $\mathrm{Fe}_{3} \mathrm{O}_{4}$ results in epitaxial growth of magnetite. Symmetrical $\theta / 2 \theta$ x-ray scans showed the presence of the $(4,0,0)_{C}$ reflection and the $(4,4,0)_{C}$ reflection from the $\mathrm{Fe}_{3} \mathrm{O}_{4}$ films near the $(2,0,0)$ and $(2,2,0)$ Bragg peaks from the MgO substrates, respectively. This confirmed the expected out of plane orientations, $\mathrm{Fe}_{3} \mathrm{O}_{4}[100]|| \mathrm{MgO}[100]$ and $\mathrm{Fe}_{3} \mathrm{O}_{4}[110]|| \mathrm{MgO}[110]$. The rocking curve width at half maximum for all films is around 0.02 degrees demonstrating their high crystalline quality and low mosaic spread. Magnetic measurements showed a sharp Verwey transition at $T_{V}=121 \mathrm{~K}$ (Orna et al., 2010), which also evidences that the films exhibit a first-order Verwey transition.

RXS measurements were performed at the Fe K-edge on the ID20 beam line (Paolasini et al., 2007) at the European Synchrotron Radiation Facility (Grenoble, France). The x-ray beam wavelength was selected by a $\mathrm{Si}(111)$ double crystal monochromator (energy resolution of 0.8 $\mathrm{eV}$ ) and the polarization of the scattered radiation was analyzed by means of a MgO (222) crystal. The experiment was carried out using a four-circle diffractometer in vertical scattering geometry. A closed He Displex cryostat with Be domes was used to cool the samples down to $10 \mathrm{~K}$. In order to minimize the number of crystallographic domains, a magnetic field was applied along one of the $\langle 001\rangle_{C}$ axes to uniquely define the monoclinic c axis on cooling through $\mathrm{T}_{\mathrm{V}}$. Multiple-scattering events were washed out by acquiring energy scans at different azimuthal $\phi$ angles (orientations of the sample respect to the incident linear polarization). RXS data presented in this paper for single crystals have been already corrected for absorption by using the linear absorption coefficient obtained from a transmission $\mathrm{x}$-ray absorption spectroscopy measurement. In the case of the thin films, we have measured the intensity of the $(2,0,0)$ reflection from the MgO substrate across the Fe K-edge. No attenuation of this substrate reflection was found, which guarantees that RXS data from the thin films are not affected by self-absorption effects. Finally, in order to express the experimental scattered intensity in absolute squared electron units, we normalized this intensity at energies far away from the absorption edge to the square of the non-resonant structure factor fixed by the structural model of displacements.

\section{Results and Data analysis}

We have measured two types of reflections: (I) reflections that are sensitive to the possible charge modulations found compatible with the $P 2 / C$ and/or $C 2 / C$ models (the anomalous scattering factor can be approximated by a scalar) and (II) reflections that are forbidden by symmetry in either the $P 2 / C$ or $C 2 / C$ space groups (ATS reflections coming from the anisotropy of the anomalous scattering factor). The sensitivity of reflections to a charge 
modulation or anisotropy is determined by their structure factor. This must contain differences between the atomic scattering factors of crystallographically non-equivalent (charge) or equivalent (anisotropy) Fe atoms. Therefore, we have calculated the structure factors for those reflections allowed in both $P 2 / c$ - and $C 2 / c$-models first using the simpler $P 2 / C$ cell, as it averages the displacements observed in the $C 2 / C$ one. Only reflections with a null structure factor in the $P 2 / C$ space group have been calculated using the $C 2 / C$ refinement (Blasco et al., 2011) with two non-equivalent tetrahedral (A1 and A2) sites and ten nonequivalent octahedral (B1a, B1b, B1b', B2a, B2a', B2b, B3, B3', B4 and B4') sites (figure 1). We have followed the notation of reference (Wright et al., 2001; Wright et al., 2002) for the sake of comparison and quotation marks differentiate equivalent sites in the $\mathrm{P} 2 / \mathrm{C}$ model. Moreover, B1a and B1b, and, B2a and B2b pairs are almost equivalent through the Pmca constraints. When the letters are omitted, we refer to both a and b sites simultaneously.

\subsection{Reflections sensitive to the charge disproportion between B1 and B2 sites}

We will start with reflections that are sensitive to the differences between B1 and B2 sites. These reflections are $(-h, h, 0)_{\mathrm{C}},(0,0, l)_{\mathrm{C}}$ and $(-4,4, l)_{\mathrm{C}}$ with $h$ and $l$ odd.

The energy dependence of the intensity close to the Fe K-edge for a number of these superlattice reflections in the $\sigma-\sigma^{\prime}$ channel is shown in Figs. 2(a), 2(b) and 2(c), respectively. Single crystal data corrected from self-absorption are compared to the thin films. The spectral line shape is very similar for both single crystals and thin films. We note that the overall shape reported for the $(-4,4, l)_{\mathrm{C}}$ reflections qualitatively agrees with data already published (Nazarenko et al., 2006). Reflections with $h=1$ or 5 and $l=1$ or 5 show an enhancement of the scattered intensity near the energy of the absorption $\mathrm{K}$ edge with two resonant peaks at about $7124.5 \mathrm{eV}$ and $7129.5 \mathrm{eV}$, respectively. On the other hand, reflections with $h=3$ or $l=3$ show a decrease of the resonant scattered signal at roughly the same energies. Generally, the observation of either peaks or valleys at the absorption threshold is indicative of a charge disproportion between the involved iron atoms.

Their structure factor $F(Q, E)$ in the $P 2 / c$ model is given by the following general expression

$$
\begin{aligned}
& F(Q, E)=\left[F_{O, F e}(Q)+a f_{A}^{\prime}+b f_{B}^{\prime}+c\left(f_{A 1}^{\prime}-f_{A 2}^{\prime}\right)+d\left(f_{B 1}^{\prime}-f_{B 2}^{\prime}\right)\right] \\
& +i\left[a f_{A}^{\prime \prime}+b f_{B}^{\prime \prime}+c\left(f_{A 1}^{\prime \prime}-f_{A 2}^{\prime \prime}\right)+d\left(f_{B 1}^{\prime \prime}-f_{B 2}^{\prime \prime}\right)\right]
\end{aligned}
$$

Here, $F_{O, F e}(Q)$ is the energy-independent (Thomson) contribution of $\mathrm{O}$ and $\mathrm{Fe}$ atoms to the

structure factor and $f_{A}^{\prime}+i f_{A}^{\prime \prime}$ and $f_{B}^{\prime}+i f_{B}^{\prime \prime}$ correspond to the energy-dependent contributions arising from the average of non-ordered tetrahedral and octahedral Fe atoms, respectively. Parameters $a, b, c$ and $d$ are related to the atomic displacements of tetrahedral (A1 and A2) and octahedral (B1, B2, B3, and B4) Fe atoms. Clearly, a resonance occurs due 
to the contrast between the anomalous scattering factors of either B1-B2 octahedral sites and/or A1-A2 tetrahedral sites, suggesting possible charge disproportions between each pair of atoms. The sensitivity to those charge disproportions will depend on the weight of the nonresonant term with respect to the resonant terms. Indeed, the values of $a$ and $b$ are small respect to those of $c$ and $d$. To illustrate this fact, we can express numerically the structure factors of $(0,0, l)_{\mathrm{C}}$ reflections with $l=1,3,5$ and 7 :

$$
\begin{aligned}
& F_{001}=\left[-4.6-0.1 f_{A}+0.3 f_{B}+2.8\left(f_{A 1}-f_{A 2}\right)+4\left(f_{B 1}-f_{B 2}\right)\right] \\
& F_{003}=\left[10-0.3 f_{A}-0.8 f_{B}-2.7\left(f_{A 1}-f_{A 2}\right)+4\left(f_{B 1}-f_{B 2}\right)\right] \\
& F_{005}=\left[-10+0.5 f_{A}+1.3 f_{B}-2.6\left(f_{A 1}-f_{A 2}\right)+4\left(f_{B 1}-f_{B 2}\right)\right] \\
& F_{007}=\left[11+0.6 f_{A}-1.8 f_{B}+2.5\left(f_{A 1}-f_{A 2}\right)+4\left(f_{B 1}-f_{B 2}\right)\right]
\end{aligned}
$$

It is also clear from the inspection of these structure factors that the non-resonant scattering term due to the atomic displacements change sign depending on the $l$ index (see for example $(0,0,3)_{\mathrm{C}}$ and $\left.(0,0,5)_{\mathrm{C}}\right)$. The fact that this Thomson contribution is either in phase or out of phase with the resonant term as a function of $\vec{Q}$ (Blasco et al., 2008a) is reflected in the occurrence of either a peak or a valley at the absorption threshold. This fact explains quite well the different energy line shapes observed within this group of reflections (Fig. 2). The same behavior has been deduced for the other two series of reflections. It is noteworthy that this analysis is not modified when the $C 2 / c$-model is applied. The only difference in the above expressions is that the A1 and A2 sites each represent an average of 2 independent sites, whereas the B1 and B2 sites each represent the average of 3 non-equivalent sites.

We have also measured some of these reflections at different azimuthal angles. Figure 3 indicates that neither the $(0,0,5)_{\mathrm{C}}$ nor the $(-3,3,0)_{\mathrm{C}}$ show a strong angular dependence of either the intensity or the shape of the spectra in the $\sigma-\sigma$ ' channel. We can confirm from this qualitative analysis the existence of a charge segregation between octahedral iron atoms located at the B1 and B2 sites. However, it seems to be rather small, as we (Subías et al., 2004a) and other authors (Nazarenko et al., 2006; Joly et al., 2008) have previously stated.

The polarization analysis shows a small $\sigma-\pi$ ' contribution for $(-h, h, 0)_{\mathrm{C}}$ and $(-4,4, l)_{\mathrm{C}}$ reflections, which is not observed for $(0,0, l)_{\mathrm{C}}$ ones. Figure 4 compares the energy dependence of the intensity for the $(-1,1,0)_{\mathrm{C}}$ reflection in the two polarization channels at $\phi=0^{\circ}$. In contrast to the $\sigma-\sigma^{\prime}$ contribution, for which the intensity is independent of the azimuthal angle, the $\sigma-\pi$ ' resonant scattering shows a characteristic oscillation with $\pi$-period (Fig. 4, inset), varying as $\cos ^{2} \phi$. The maximum intensity at $\phi=0$ corresponds to a configuration in which the $c$ axis is perpendicular to the diffraction plane. The observation of a signal in this rotated polarization channel with a clear azimuthal angle dependence reveals the anisotropy of the Fe atoms at the B1 and/or B2 sites. Because magnetite presents crystal twinning 
perpendicular to the monoclinic $c$-axis below the Verwey transition, the $(-1,1,0)_{\mathrm{C}}$ reflection is observed for one crystallographic domain, while the $(1,1,0)_{\mathrm{C}}$ is present in the neighbour domain. The experimental geometry with the monoclinic $a_{\mathrm{m}}\left(b_{\mathrm{m}}\right)$ axis perpendicular to the sample surface does not allow us to differentiate both reflections. We have considered the two domains to deduce the intensities in the two polarization channels. For the $(-1,1,0)_{\mathrm{C}}$ reflection, the symmetry operations in the $P 2 / C$-model make the structure factor to be diagonal of the form

$F_{-110}=\left(\begin{array}{ccc}F_{x x} & 0 & 0 \\ 0 & F_{y y} & 0 \\ 0 & 0 & F_{z z}\end{array}\right)$

being $\quad F_{x x}=-0.12-0.4 f_{x x}^{B 3}+0.08 f_{x x}^{B 4}+3.997\left(f_{x x}^{A 1}-f_{x x}^{A 2}\right)-3.995\left(f_{x x}^{B 1}-f_{x x}^{B 2}\right)$

and similar expressions are found for $F_{\mathrm{yy}}$ and $F_{\mathrm{zz}}$. We deduce then that

$I_{\sigma-\sigma^{\prime}}=\left|F_{x x} \sin \phi^{2}+F_{z z} \cos \phi^{2}\right|^{2}$

and

$$
I_{\sigma-\pi^{\prime}}=\left|\left(F_{x x}-F_{z z}\right) \sin \theta \sin 2 \phi\right|^{2}
$$

Since we have not observed any azimuthal dependence of the $\sigma-\sigma$ ' intensity for the ($3,3,0)_{\mathrm{C}}$ reflection (see inset in Fig. $3 \mathrm{~b}$ ), $F_{\mathrm{xx}}=F_{\mathrm{zz}}$ and, consequently, $I_{\sigma-\pi^{\prime}}$ is zero. We now check the $(1,1,0)_{\mathrm{C}}$ domain. The structure factor for this reflection is strictly zero in the $P 2 / c$ model (Wright et al., 2001; Wright et al., 2002), but it is very weak (and non-resonant) in the $C 2 / c$-model. By using the symmetry operations of the $P 2 / C$-model, we find that for the $(1,1$, $0)_{\mathrm{C}}$ reflection the structure factor is a tensor of the form

$$
F_{110}=\left(\begin{array}{ccc}
0 & 0 & F_{x z} \\
0 & 0 & 0 \\
F_{x z} & 0 & 0
\end{array}\right)
$$

Contributions to the $F_{x z}$ component can be originated by either the B1 and/or the B2 sites. It follows then

$$
I_{\sigma-\sigma^{\prime}}=0
$$

and

$$
I_{\sigma-\pi^{\prime}}=\left|F_{x z} \cos \theta \cos \phi\right|^{2}
$$

Therefore, the experimental azimuthal behavior found in the $\sigma-\pi$ ' resonant scattering (inset of Fig. 4) is only due to this domain. The occurrence of ATS reflections is related to the presence of translational symmetry elements (screw axis and/or glide planes) in the space group. In this particular case, it is worth explaining the symmetry implications step by step. 
Our analysis is done in the Pmca-model, as the P2/c-model maintains the Pmca constraints. The B1 sites have local inversion symmetry and the two groups of two atoms that are in antiphase are related by a screw axis, while the $\mathrm{B} 2$ sites include a $C_{2 y}$ site-symmetry and the two groups of atoms that are in antiphase are related by an $a$ glide plane. When the symmetry is reduced to the $P 2 / C$-model, the two groups that are in antiphase correspond to either B1a and B1b or B2a and B2b, respectively. Finally, the total contribution of A1, A2, B3 and B4 is equal to zero since they have a local $m_{x}$-symmetry, but the two groups that are in antiphase are related by the inversion symmetry. The fact that this $F_{x z}$ term becomes allowed clearly indicates that the B1 (and/or B2) iron atoms must be anisotropic and the anomalous scattering factor tensor changes its orientation under the screw-axis (or the glide-plane) symmetry operations so that the tensors of the two groups of B1 (and/or B2) symmetry related atoms cannot compensate each other.

\subsection{Reflections sensitive to the charge disproportion between B3 and B4 sites}

Half-integer reflections such as $(h, k, l / 2)_{\mathrm{C}}$ with $l$ odd have been investigated to unambiguously determine if there is a charge segregation between the B3 and B4 sites. In figure 5, we show the energy dependent spectra for some of these reflections. We distinguish three groups: (a) reflections with $h, k=$ even like $(-4,4,1 / 2)_{\mathrm{C}}$ or $(-4,2,1 / 2)_{\mathrm{C}}$, which have been previously considered to be a signature of the CO between B3 and B4 sites; (b) reflections with $h, k=$ odd like $(-3,3, l / 2)_{\mathrm{C}}$, whose structure factor actually reflects differences between the atomic anomalous scattering factors of the B3 and B4 sites and (c) reflections with $h+k$ $=$ odd like $(2,1,1 / 2)_{\mathrm{C}}$ and $(-5,0,1 / 2)_{\mathrm{C}}$. This last group includes reflections that are forbidden in the $P / 2 c$ symmetry but allowed in the $C 2 / c$ space group.

The analysis of these reflections will provide us information on a possible charge disproportion between formally equivalent B3 (or B4) atoms in P2/c-model that are split into non-equivalent sites in $\mathrm{C} 2 / \mathrm{c}$-model. None of the measured reflections within the first group (figure 5a) shows any strong enhancement or drop in the intensity that can be directly related to a resonance. Moreover, the evaluation of the structure factors for the $(-4,4,1 / 2)_{\mathrm{C}}$ reflections indicates that they are not sensitive to differences in scattering factor among either octahedral or tetrahedral sites. The only exception is the $(-4,4,5 / 2)_{\mathrm{C}}$ reflection, which shows an enhancement of intensity at $7.13 \mathrm{keV}$. This energy dependence is explained by the fact that the structure factor is sensitive to the difference in scattering factor between the Fe in the tetrahedron and that of the Fe in the octahedron. Regarding the second group, superlattice reflections with $l=1$ and 5 recorded in this work (figure $5 \mathrm{~b}$ ) do not show any strong maximum (or minimum) at the Fe K-edge, either. Moreover, both sets of half-integer reflections display the same global energy line shape. However, the structure factor of the $(-3,3,1 / 2)_{\mathrm{C}}$ and $(-4,2$, $1 / 2)_{\mathrm{C}}$ reflections in the $P 2 / c$-model can be expressed by 


$$
\begin{aligned}
& F(Q, E)=\left[F_{O, F e}(Q)+a f_{A}^{\prime}+b f_{B}^{\prime}+c\left(f_{A 1}^{\prime}-f_{A 2}^{\prime}\right)+d\left(f_{B 4}^{\prime}-f_{B 3}^{\prime}\right)\right\rfloor \\
& +i\left[a f_{A}^{\prime \prime}+b f_{B}^{\prime \prime}+c\left(f_{A 1}^{\prime \prime}-f_{A 2}^{\prime \prime}\right)+d\left(f_{B 4}^{\prime \prime}-f_{B 3}^{\prime \prime}\right)\right]
\end{aligned}
$$

and the values of $a, b$ and $c$ parameters are small with respect to that of $d \sim 3$. The above reflections should be, then, suitable to reflect any signature of the $\mathrm{Fe}_{\mathrm{B} 3}-\mathrm{Fe}_{\mathrm{B} 4}$ charge disproportion. Therefore, we can conclude that the supposedly different B3-site and B4-site iron atoms in the $P 2 / C$ model do not actually show any significant charge disproportion.

The third group of reflections is only allowed in the $C / 2 c$ symmetry. A two-peak resonance is observed at 7.124 and $7.130 \mathrm{keV}$ with almost no intensity outside this energy range for some of these reflections such as the $(2,1,1 / 2)_{\mathrm{C}}$ and $(-5,0,1 / 2)_{\mathrm{C}}$ shown in figure $5 \mathrm{c}$. The structure factor for these reflections can be expressed by

$$
\begin{aligned}
& F_{-211 / 2}(Q, E) \infty\left[F_{O}(Q)+1.4 \Delta f_{B 3}+1.4 \Delta f_{B 4}-\Delta f_{B 1}\right] \\
& \left.F_{-501 / 2}(Q, E) \infty\left[F_{O}(Q)+0.15 f_{A}+0.1 f_{B}+1.6 \Delta f_{B 3}+1.3 \Delta f_{B 4}+\Delta f_{B 1}\right)\right]
\end{aligned}
$$

being $\Delta f_{B 1}, \Delta f_{B 3}$ and $\Delta f_{B 4}$ the difference between the two non-equivalent B1b, B3 and B4 sites in the $C 2 / C$-model. This result indicates a small charge disproportion between distinct B3 (B4 and/or B1b) sites, which qualitatively agrees with the crystal structure obtained from the $C 2 / C$ space group (Blasco et al., 2011).

\subsection{Half-integer ATS reflections sensitive to local anisotropies}

We have extensively studied the anisotropy between crystallographically equivalent $\mathrm{Fe}$ atoms by measuring the $(0,0, l / 2)_{\mathrm{C}}$ reflections with $l=5,7,9$ and 11 . Their energy dependences recorded in the $\sigma-\pi$ ' channel are shown in figure 6 . The two data sets collected either on the single-crystal after absorption correction or on the thin film are self-consistent. The energy scans of the $(0,0,5 / 2)_{\mathrm{C}}$ reflection in the $\sigma-\sigma^{\prime}$ and $\sigma-\pi^{\prime}$ polarization channels are compared for the single-crystal A in figure 6(a). These reflections are only seen in the rotated polarization channel $\left(\sigma-\pi^{\prime}\right)$ on resonance. The null intensity of these reflections in the $\sigma-\sigma$ ' channel agrees with the presence of a $c$-glide plane in the low temperature crystal structure of magnetite. Therefore, the observed energy and polarization behavior indicate that these are glide-plane forbidden ATS reflections. No polarization analysis was performed in our earlier work (Subías et al., 2004a), which is most likely the reason why we were unable to detect these reflections in previous scans along the $l$ index. $(0,0,5 / 2)_{\mathrm{C}}$ and $(0,0,11 / 2)_{\mathrm{C}}$ forbidden reflections have a very similar energy dependence. In contrast, $(0,0,7 / 2)_{C}$ and $(0,0,9 / 2)_{C}$ forbidden reflections show a different energy profile, although they are very similar to each other. Moreover, the latter show a two-peaked resonant feature.

To explain these differences in the energy line shape depending on the $l$-index, we have calculated the structure factor of these ATS reflections that is a symmetric tensor in which the diagonal components become zero. Using the $C 2 / c$ structure, and taking into 
account that the atomic scattering tensor at each atomic site is invariant under its own site symmetry and related to the equivalent Wyckoff positions through the space group symmetry operations, the only off-diagonal elements of the final structure factor that may contribute are the $f_{\mathrm{xy}}$ and the $f_{\mathrm{yz}}$ components with allowed contributions from all Fe sites except B2. We note that $f_{\mathrm{xy}}$ is forbidden in the Pmca undistorted structure with $\beta=90^{\circ}$. As the monoclinic distortion from the orthorhombic structure is very small, we can neglect the $f_{\mathrm{xy}}$ component with respect to $f_{\mathrm{yz}}$, and the resonant scattering can be modeled in the Pmca-model more easily. Therefore, the $\sigma-\pi^{\prime}$ intensity for the selected $(0,0,5 / 2)_{\mathrm{C}},(0,0,7 / 2)_{\mathrm{C}},(0,0,9 / 2)_{\mathrm{C}}$ and $(0,0$, $11 / 2)_{\mathrm{C}}$ reflections in the latter model is given by

$$
\begin{aligned}
& I_{(005 / 2)}=16 \sin ^{2} \varphi \cos ^{2} \theta\left[-\sin \frac{\pi}{8} f_{y z}^{A 1}+\cos \frac{\pi}{8} f_{y z}^{A 2}+f_{y z}^{B 1}+\sin \frac{\pi}{4}\left(f_{y z}^{B 3}+f_{y z}^{B 4}\right)\right]^{2} \\
& I_{(007 / 2)}=16 \sin ^{2} \varphi \cos ^{2} \theta\left[-\cos \frac{\pi}{8} f_{y z}^{A 1}-\sin \frac{\pi}{8} f_{y z}^{A 2}+f_{y z}^{B 1}-\sin \frac{\pi}{4}\left(f_{y z}^{B 3}+f_{y z}^{B 4}\right)\right]^{2} \\
& I_{(009 / 2)}=16 \sin ^{2} \varphi \cos ^{2} \theta\left[-\cos \frac{\pi}{8} f_{y z}^{A 1}-\sin \frac{\pi}{8} f_{y z}^{A 2}+f_{y z}^{B 1}+\sin \frac{\pi}{4}\left(f_{y z}^{B 3}+f_{y z}^{B 4}\right)\right]^{2} \\
& I_{(0011 / 2)}=16 \sin ^{2} \varphi \cos ^{2} \theta\left[-\sin \frac{\pi}{8} f_{y z}^{A 1}+\cos \frac{\pi}{8} f_{y z}^{A 2}+f_{y z}^{B 1}-\sin \frac{\pi}{4}\left(f_{y z}^{B 3}+f_{y z}^{B 4}\right)\right]^{2}
\end{aligned}
$$

8)

We can extract several conclusions from the simple inspection of the above expressions. If tetrahedral sites were not anisotropic, the energy variation of the intensity of the $(0,0,5 / 2)_{\mathrm{C}}$ reflection would be identical to that of the $(0,0,9 / 2)_{\mathrm{C}}$ reflection. The same identity in the energy scans would apply to the $(0,0,7 / 2)_{C}$ and $(0,0,11 / 2)_{C}$ reflections. This is in contrast to the experimental results shown in figure 6. On the other hand, if octahedral B3 and B4 sites were not anisotropic, $(0,0,5 / 2)_{\mathrm{C}}$ and $(0,0,11 / 2)_{\mathrm{C}}$ reflections would have the same energy dependence whereas the energy profile of the $(0,0,7 / 2)_{\mathrm{C}}$ reflection would be identical to that of the $(0,0,11 / 2)_{\mathrm{C}}$ one. In fact, all the measured $(0,0, l / 2)_{\mathrm{C}}$ reflections show energy dependences slightly different to each other. Therefore, interference among different nonequivalent Fe sites, including tetrahedral ones, is necessary to explain the Fe K-edge resonant scattering in $(0,0, l / 2)_{\mathrm{C}} l=$ odd ATS reflections. Another characteristic property of the ATS reflections is the azimuthal dependence of the resonant intensity. However, figure 7 does not reveal any dependence of the integrated intensity of the $(0,0,7 / 2)_{\mathrm{C}}$ reflection on resonance as a function of the azimuthal angle. It is clear from the above expressions that these $(0,0, l / 2)_{C}$ reflections follow a $\pi$-periodicity, however masked by the presence of both, $[100]_{C}$ and $[010]_{C}$ oriented domains at the sample surface.

Moreover, the appearance of different local distortions around these Fe sites has been proved to occur at the structural transition by our x-ray diffraction data and thus, the electronic anisotropy arising from these crystal distortions is sufficient to explain the origin of the observed resonant signals. 


\subsection{Temperature evolution of charge and local anisotropy modulations}

Figure 8 compares the temperature dependence of the $(0,0,1)_{\mathrm{C}}$ superlattice reflection and the $(0,0,7 / 2)_{\mathrm{C}}$ ATS reflection in the single crystal and the thin film on resonance. The two types of reflections occur simultaneously below $\mathrm{T}_{\mathrm{V}}$, at $\sim 120 \mathrm{~K}$ in the thin film and at about $123 \mathrm{~K}$ in the single crystal. The temperature values agree with those reported from the macroscopic magnetic measurements. A sharp jump is observed at $T_{V}$ in the temperature dependence of the $(0,0,1)_{\mathrm{C}}$ reflections, whereas the $(0,0,7 / 2)_{\mathrm{C}}$ reflections continuously decrease down to a temperature close to $T_{V}$, where a more drastic decay is observed. This confirms our previous result that the structural distortions and the associated charge and anisotropies modulations are concomitant with the first order Verwey transition, reflecting a common origin not only for single crystals (García et al., 2009), but also for thin films.

\section{Simulations and charge disproportions}

Simulations and fits of the experimental data are needed to precisely determine the size of the associated charge and/or anisotropy modulations. Previous to any modeling of the RXS, we reliably determined the atomic anomalous scattering factor of the iron atoms resolved in the two sites, tetrahedral and octahedral. With this purpose, we measured selected Bragg reflections characteristic of the cubic phase at room temperature in the thin film for which contributions to the structure factor from one Fe site is null, leaving contributions from only the other site. We chose the $(2,2,0)_{C}$ and $(2,2,2)_{C}$ reflections since the former is sensitive to the tetrahedral scattering factor, while the latter has only a contribution from the octahedral scattering factor. In addition, the $(4,4,0)_{\mathrm{C}}$ and $(4,4,4)_{\mathrm{C}}$ reflections, which originate from contributions from both sites were also measured to check the reliability of the extracted sitespecific scattering factors. Thin film B was used in order to avoid absorption problems. Figure 9 shows the experimental energy-scans for the tetrahedral, octahedral and mixed reflections at the Fe K-edge. By neglecting second-order terms in the analysis of the diffracted intensity (Proietti et al., 1999), the real parts of the tetrahedral and octahedral anomalous scattering factors can be extracted directly out from the experimental spectra of the $(2,2,0)_{\mathrm{C}}$ and $(2,2,2)_{\mathrm{C}}$ reflections, respectively. The imaginary parts are then obtained from the Kramers-Kronig relation. We show in figure 10 the real $\left(f^{\prime}\right)$ and imaginary $\left(f^{\prime}\right)$ parts of the anomalous scattering factors experimentally obtained for the tetrahedral and octahedral Fe atoms in magnetite. The difference between the first inflection point energies of the tetrahedral and octahedral sites is approximately $1.0 \pm 0.2 \mathrm{eV}$. A quantitative check of the extracted anomalous scattering amplitudes is also shown in figure 9, in which it is clear that calculated energy-scans match quite well the profile of the experimental data for the mixed reflections. We have also checked that the x-ray absorption spectrum of magnetite measured 
in transmission with a high energy resolution (Subías et al., 2005) is well reproduced by the weighted addition 1:2 of the tetrahedral and octahedral imaginary parts.

The next step is the fit of the experimental energy dependence of the x-ray intensity for the different groups of resonant reflections to $I \propto\left|F^{*}(Q, E) F(Q, E)\right|$, where $F(Q, E)$ is the structure factor as given in the previous section.

\subsection{Charge disproportion on the B1 and B2 sites}

We performed fits to the experimental energy scans ( $\sigma-\sigma^{\prime}$ channel) of reflections ( $h, h$, $0)_{\mathrm{C}},(0,0, l)_{\mathrm{C}}$ and $(-4,4, l)_{\mathrm{C}}$ with $h$ and $l$ odd to quantify the charge disproportion between B1 and B2 sites. We fixed the atomic positions in the P2/C-model, and we only refined the magnitude of the charge disproportion. For this purpose, we used the linear dependence of the Fe valence as a function of the K-edge shift. Thus, a rigid energy shift of $\pm \delta \mathrm{E} e \mathrm{~V}$ has been applied to the scattering factors experimentally obtained for either the tetrahedral or octahedral Fe atoms. This approximation is valid because the local structure around tetrahedral and octahedral sites is only slightly modified at the structural transition. The best results for the single crystals are shown in figure 11 . We find that the refined pattern of displacements permits to reproduce quite well all the measured RXS spectra for an applied chemical shift of $0.7 \pm 0.1 \mathrm{eV}$ between the scattering factors of the Fe atoms at B1 and B2 sites. This K-edge shift corresponds to a charge disproportion of $0.20 \pm 0.05 \mathrm{e}^{-}$. This result is very close to previous estimations by RXS (Subías et al., 2004a; Nazarenko et al., 2006; Joly et al., 2008), and also agrees with a small charge difference resulting from the bond valence sums applied to either the $P 2 / C$ - (Wright et al., 2001; Wright et al., 2002) or C2/C-models (Blasco et al., 2011). We also find a slightly better agreement when a small charge disproportion between Fe atoms at A1 and A2 sites is considered, especially for the $(0,0,1)_{C}$, $(-1,1,0)_{\mathrm{C}}$ and $(-4,4,1)_{\mathrm{C}}$ reflections. The effect of charge disproportions is stronger for those reflections due to the weak amplitude of their non-resonant scattering terms. We have set a maximum value for the charge disproportion on these tetrahedral sites of $0.1 \mathrm{e}-$. From all these simulations, we can conclude a small charge disproportion along the $c$-axis direction between the octahedral B1 and B2 sites, which is driven by the geometrical distortions occurring at the structural transition.

\subsection{Charge disproportion on the B3 and B4 sites}

The effect of a charge disproportion between the B3 and B4 sites is expected to be contained in half-integer reflections such as $(-3,3,1 / 2)_{\mathrm{C}}$ or $(-4,2,1 / 2)_{\mathrm{C}}$. Because no effect in their energy-scans is clearly seen in figures 5(a) and 5(b), this charge disproportion, if exists, is expected to be very small. Nevertheless, we have quantified its magnitude using the 
weakest reflection, i.e. $(-4,2,1 / 2)_{C}$. Figure 12 compares the results of three models, (a) no charge disproportion; (b) the same charge disproportion as the one found out between B1 and B2 sites, and (c) a charge disproportion with half the magnitude of the previous disproportion. From these simulations, we can rule out a significant charge disproportion between the B3 and B4 sites.

In addition, our x-ray diffraction refinement in the $C 2 / c$ crystal symmetry gives a split of the octahedral B1b, B2a, B3 and B4 sites into two non-equivalent sites each one, resulting in a charge difference between averaged B3 and B4 sites of 0.09 $\pm 0.03 \mathrm{e}^{-}$(Blasco et al., 2011), which can be set as the high limit for any charge disproportion being compatible with our RXS data. Moreover, a larger charge difference between the two B3 sites (or B4 or B1b sites) is found that is of the same order of magnitude as the B3-B4 charge disproportion deduced from the P2/C model (Wright et al., 2001; Wright et al., 2002). In order to test these new charge disproportion, we have simulated the $(2,1,1 / 2)_{\mathrm{C}}$ and $(-5,0,1 / 2)_{\mathrm{C}}$ reflections that are only permitted in the larger $C 2 / c$ cell. Figure 13 compares the best-fit simulated intensity and the corresponding experiment. From our simulations, the charge disproportion in the three different pairs of iron atoms (B1b-B1b'), (B3-B3') and (B4-B4') is $0.15 \pm 0.05 \mathrm{e}^{-}$. This result compares quite well within the uncertainty with the $0.17 \pm 0.03 \mathrm{e}^{-}$given by the $\mathrm{x}$-ray diffraction refinement (Blasco et al., 2011).

Again, the appearance of these charge disproportions is intimately correlated with the structural distortions, which take place from the cubic to the monoclinic $C 2 / c$ structure.

\section{Discussion and conclusions}

We have performed an exhaustive experimental study of the Verwey transition in magnetite by RXS at the Fe K-edge on high quality single-crystals and thin films. From these results and earlier RXS works (Kanazawa et al., 2002; García et al., 2000; García et al., 2001; Subías et al., 2004a; Nazarenko et al., 2006; Joly et al., 2008; García et al., 2009) in combination with the recent $\mathrm{x}$-ray powder refinement of the low temperature crystallographic phase of magnetite in the $C 2 / c$-model (Blasco et al., 2011), we provide a realistic description of the structural changes, charge disproportions and local anisotropies that take place at the Verwey transition in this material.

The first conclusion deals with the long standing controversy of whether CO occurs at the octahedral iron sites or not. First RXS studies of the forbidden $(0,0,4 n+2)_{C}$ reflections completely discarded a CO of the Verwey type (Kanazawa et al., 2002; García et al., 2000; García et al., 2001) and established a maximum charge disproportion of 0.25 electrons. Later, other models consistent with the P2/c refinement of Wright et al. (Wright et al., 2001; Wright et al., 2002) and that do not fulfill the Anderson criterion, proposed a non-integer bimodal $\mathrm{CO}$, i.e. they divided the octahedral B iron atoms into two equal groups with different valence 
states, $\mathrm{Fe}^{+2.5-\delta}$ and $\mathrm{Fe}^{+2.5+\delta}$, with a very small value of $\delta$. The key point of the present study is to clearly show that the distribution of non-integer valences among the different octahedral $\mathrm{Fe}$ sites is not bimodal either. We start from the crystallographic description within the $P / 2 c$ model, which is a good average approach because further atomic displacements are small. We reported on resonances at the $(0,0, l)_{\mathrm{C}}$ and $(-h, h, 0)_{\mathrm{C}}$ with $l$ and $h$ odd reflections allowed in this $\mathrm{P} 2 / \mathrm{c}$-model, that demonstrates the existence of a charge density wave propagating along the $\left[\begin{array}{lll}0 & 0 & 1\end{array}\right]_{\mathrm{C}}$ and $\left[\begin{array}{lll}1 & 1 & 0\end{array}\right]_{\mathrm{C}}$ directions with an associated charge disproportion of $0.20 \pm 0.05$ electrons between the averaged B1 and B2 sites, in agreement with the results previously reported by Wright et al. (Wright et al., 2001; Wright et al., 2002) and Nazarenko et al. (Nazarenko et al., 2006) by using the same crystallographic model. The main discrepancy between the P2/c-model and our RXS results concerns the charge disproportion between B3 and B4. We did not find any resonance in the reflections sensitive to this ordering. Therefore, the charge disproportion between the averaged B3 and B4 sites should be very small, lower than 0.1 electrons. The absence of charge disproportion on these octahedral sites is in better agreement with the $C 2 / c$-model (Blasco et al., 2011). In fact, the four iron atoms at B3 and B4 sites are split into two different crystallographic sites each one, giving rise to multiple $\mathrm{Fe}$ valences ranging between 2.53 and 2.84 that make the charge disproportion between the average B3 and B4 sites to be strongly reduced. Moreover, we found an appreciable segregation of about 0.15 electrons between the two non-equivalent B3 and B3' (or B4 and B4') atoms, which is fully compatible with the $C 2 / c$ symmetry. We note that B1 atoms are also split in the $C 2 / c$ symmetry and a charge disproportion among them has been deduced too. Finally, it is noteworthy that in the $C 2 / c$-model, the condition that the same average charge is contained in the B3+B4 and B1+B2 planes deduced from the study of the $(0,0,4 n+2)_{C}$ resonant reflections (García et al., 2001) is also satisfied. Therefore, due to the complexity of the $C 2 / c$ distortion pattern in the low temperature phase, no bimodal charge disproportion is found opposite to the classical $\mathrm{Fe}^{2+}$-like $-\mathrm{Fe}^{3+}$-like $\mathrm{CO}$ model.

The second conclusion deals with the anisotropy of the octahedral $\mathrm{B}$ atoms and the correlation with the OO. We recall here that RXS of the forbidden $(0,0,4 n+2)_{C}$ reflections in magnetite (Kanazawa et al., 2002; García et al., 2000; García et al., 2001) and other substituted spinel ferrites (Subías et al., 2004b) demonstrated a strong anisotropy (trigonal distortion) of the octahedral $\mathrm{B}$ sites, which remains unaltered below $\mathrm{T}_{\mathrm{V}}$. This implies that, independently of the local distortions and/or charge segregation occurring in the low temperature phase, all the B atoms maintain this trigonal anisotropy. However, to date, none of the proposed CO models has considered this strong trigonal anisotropy for the octahedral atoms. Instead, they proposed other $\mathrm{OO}$ associated to the $\mathrm{t}_{2 \mathrm{~g}}$ occupancy of the $\mathrm{Fe}^{2+}$-like atoms. We have also analyzed our experimental RXS results taking into account the trigonal 
anisotropy of the B sites (García et al., 2001). The fact that $(2,0,0)_{C},(0,2,0)_{C}$ and $(0,0,2)_{C}$ ATS reflections only show the trigonal anisotropy together with the pure $\sigma-\sigma$ ' character of the superlattice $(0,0,1)_{\mathrm{C}}$ and $(-1,1,0)_{\mathrm{C}}$ reflections demonstrates the equivalence of trigonal anisotropies at the B1, B2, B3 and B4 sites in the P2/c-model. This means that the averaged anisotropy of the B-atoms is identical independently of the charge disproportion between B1 and B2 or between B3 and B4 sites. Furthermore, we have observed a $\sigma-\pi^{\prime}$ contribution to the $(1,1,0)_{\mathrm{C}}$ and $(0,0, l / 2)_{\mathrm{C}}$ reflections. We have shown that these reflections are also ATS as a result of the structural transition. The contribution to the $(1,1,0)_{\mathrm{C}}$ comes from an ordered anisotropy between the two B1a and B1b (and/or B2a - B2b) atoms in the P2/c-model despite the averaged B1-site and B2-site anisotropies are identical. This anisotropy results from a symmetry breaking of the screw axis at the B1 sites (Pmca-model). Regarding $(0,0, l / 2)_{\mathrm{C}}$ reflections, the $\sigma-\pi^{\prime}$ resonance comes from an ordered anisotropy between the atoms at the same B sites except for B2, and it is related to the $c$-glide plane. Moreover, the shape of the energy scans is dependent on the $l$-index of the reflection, which implies that also tetrahedral A sites must be anisotropic. Following these results, it is very difficult to associate the observation of RXS at the $(0,0,1 / 2)_{\mathrm{C}}$ reflection at both the $\mathrm{Fe} \mathrm{L}_{3,2}$ and the $\mathrm{O} \mathrm{K}$ edge to a $\mathrm{t}_{2 \mathrm{~g}}$ OO on B1 and B4 sites, as it was theoretically suggested (Leonov et al., 2004; Jeng et al., 2004; Rowan et al., 2009). Moreover, such an OO does not correspond to the $\left[\begin{array}{lll}0 & 0 & 1 / 2\end{array}\right]_{\mathrm{C}}$ periodicity either. Finally, it is noteworthy that the intensity of the additional anisotropic reflections originated by the Verwey transition is about two orders of magnitude lower than that of $(0,0,4 \mathrm{n}+2)_{\mathrm{C}}$ ATS reflections. Thus, these additional anisotropies are small corrections to the trigonal one.

In summary, we have shown that the RXS behavior at the Fe K-edge of superstructure and ATS reflections in magnetite below the Verwey transition is fully compatible with the monoclinic $C 2 / c$ cell resulting from the condensation of soft phonon modes. Due to this structural transition, a charge modulation of tiny amplitude with a wave vector $(0,0,1)_{\mathrm{C}}$ and $(-1,1,0)_{\mathrm{C}}$ is stabilized. Additional minor charge modulations in the monoclinic $a-b$ plane are also present. Independently of these weak charge modulations, the strong average trigonal anisotropy of the octahedral atoms present in the cubic phase above $\mathrm{T}_{\mathrm{V}}$ does not change below $\mathrm{T}_{\mathrm{V}}$. Moreover, additional small anisotropies also occur in the low temperature phase due to structural differences between Fe atoms at the same B-site. Finally, the observation of the same behavior on two kinds of high quality samples (single crystals and epitaxial thin films) strongly supports our conclusions. The Verwey transition leads to a very complicated structural and electronic pattern, which is difficult to bring into line with a model of charge localization on $\mathrm{Fe}^{3+}$-like and $\mathrm{Fe}^{2+}$-like sites. It is better explained in terms of a charge-density wave formation at the structural first order phase transition, enlarging the band gap with a 
concomitant decrease in conductivity. Moreover, the condensation of several phonon modes leads to a wide distribution of different local environments around the octahedral iron atoms, which also definitively disproves the existence of any bimodal charge disproportion on the octahedral sites.

Figure 1 The low temperature structure of magnetite in the C2/c unit cell. Octahedral Fe B site ions are shown as red spheres and tetrahedral Fe A site ions are shown as yellow spheres. B1 and B2 site ions form separate chains parallel to the $a$ axis and B3 and B4 site ions form mixed chains parallel to the $b$ axis, stacked in layers in the order $\mathrm{B} 1, \mathrm{~B} 3+\mathrm{B} 4, \mathrm{~B} 2, \mathrm{~B} 3+\mathrm{B} 4$, connected by Fe A site ions, along the $c$ axis. B1b and B1b', B2a and B2a', B3 and B3' and B4 and B4' pairs are crystallographically equivalent in the $P 2 / c$ cell.

Figure 2 Energy-dependent scans at $10 \mathrm{~K}$ of (a) $(0,0, l)$ reflections ( $l=1,3,5$ and 7), (b) (-4, 4, l) reflections ( $(=1,3$ and 5), (c) ( $-h, h, 0)$ reflections ( $h=1$ and 3 ). Red lines represent the experimental energy dependence for the respective reflections measured in thin films and symbols refer to the bulk single crystals. Intensities have been scaled so as to fit into the graph.

Figure 3 (a) Energy dependence of the $(0,0,5)_{\sigma-\sigma}$, reflection from the single-crystal $\mathrm{A}$ at different azimuthal angles. Inset: Integrated intensity taken at $\mathrm{E}=7.125 \mathrm{keV}$ as a function of the azimuthal angle. Data have been normalized by the intensity of the $(0,0,4)$ Bragg reflection. (b) Energy dependence of the $(-3,3,0)_{\sigma-\sigma}$ reflection from the thin film $B$ at the azimuthal angles indicated in the figure. Inset: Integrated intensity taken at $\mathrm{E}=7.133 \mathrm{keV}$ as a function of the azimuthal angle.

Figure 4 Energy dependence of the $(-1,1,0)$ reflection (thin film B) in the vicinity of the Fe K-edge measured at $10 \mathrm{~K}$ and at $\phi=0^{\circ}$ in both, $\sigma-\sigma^{\prime}$ and $\sigma-\pi^{\prime}$ polarization channels. Inset: Azimuthal dependence of the $\sigma-\pi$ ' intensity on resonance.

Figure 5 (a) Energy-dependent scans of the $(-4,4, l / 2)$ reflections ( $l=$ odd) collected at $\mathrm{T}=10 \mathrm{~K}$. (b) Energy-dependent scans of the $(-3,3, l / 2)(l=$ odd $)$ and $(-4,2,1 / 2)$ reflections collected at $\mathrm{T}=10 \mathrm{~K}$. (c) Energy-dependent scans of the $(2,1,1 / 2)$ and $(-5,0,1 / 2)$ reflections only permitted in the $C 2 / c$ space group at $\mathrm{T}=10 \mathrm{~K}$. The red lines represent the experimental energy dependence measured in the thin films. Intensities have been scaled so as to fit into the graph.

Figure 6 Energy-dependent scans of $(0,0,5 / 2)(a),(0,0,7 / 2)(b),(0,0,9 / 2)(c)$ and $(0,0,11 / 2)(d)$ reflections at $10 \mathrm{~K}$ in the $\sigma-\pi$ ' channel. Lines plus open circles represent the experimental data for the single-crystal A corrected for absorption, while red solid lines represent the experimental data for the thin film A. The signal collected in the $\sigma-\sigma$ ' detection channel for the $(0,0,5 / 2)$ reflection measured in the single-crystal (lines plus closed circles) is also shown for comparison in panel (a).

Figure 7 Azimuthal behavior of the $(0,0,7 / 2)_{\sigma-\pi}$ ' reflection at $\mathrm{E}=7.125 \mathrm{keV}$. Data have been normalized by the intensity of the $(0,0,4)$ Bragg reflection. 
Figure 8 Temperature dependence of the integrated intensities of the $(0,0,1)_{\sigma-\sigma^{\prime}}$ (upper panel) and $(0,0,7 / 2)_{\sigma-\pi^{\prime}}$ (lower panel) reflections on resonance (Fe K-edge) for the single-crystal A compared to the thin film A. Intensities have been normalized to the low-temperature value for comparison purposes. Dotted lines are an eye-guide for the respective Verwey transition temperatures.

Figure 9 Experimental (circles) and calculated (solid lines) energy-dependent spectra for the (a) (2, 2, 0), (b) (2, 2, 2), (c) $(4,4,0)$ and (d) $(4,4,4)$ Bragg reflections at the Fe K-edge in the thin film B.

Figure 10 Imaginary (top) and real (bottom) parts of the anomalous scattering factor for the tetrahedral (open circles) and octahedral (closed circles) sites.

Figure 11 Comparison between simulated (solid line) and measured (circles) intensities at the Fe Kedge for a number of reflections corresponding to the $P 2 / c$ model of displacements. The simulations include charge disproportions between tetrahedral A1-A2 and octahedral B1-B2 atoms.

Figure 12 Energy variation of the simulated intensities (lines) and corresponding experiment (circles) of the $(-4,2,1 / 2)$ reflection in the thin film $B$. The three simulated curves result from (a) the $P 2 / c$ model of displacements without any charge disproportion (solid red line); (b) with charge disproportions on the octahedral B3-B4 (0.2e') atoms included (dotted green line) and (c) with the charge disproportion on the octahedral B3-B4 atoms reduced to half this value (dashed blue line).

Figure 13 Comparison between simulated (solid line) and measured (circles) intensities at the Fe Kedge for the $C 2 / c$ reflections (a) $(2,1,1 / 2)$ and (b) $(-5,0,1 / 2)$ not accounted by the $P 2 / c$ model. The simulations include charge disproportions between octahedral B1b-B1b', B3-B3' and B4-B4' atoms.

Acknowledgements The authors thank ESRF for granting beam time and for support during the experiments. We would like to thank H. Renevier and M. G. Proietti for their expert assistance in the RXS analysis and C. R. Natoli for helpful discussions. This work has been financially supported by the Spanish MICINN (FIS08-03951 and MAT2008-06567-C02 projects) and DGA (CAMRADS project).

\section{References}

Anderson, P. W. (1956). Phys. Rev. 102, 1008-1013.

Aragón, R., Buttrey, D. J., Shepherd, J. P. \& Honig, J. M., (1985). Phys. Rev. B 31, 430-436.

Bland, S. R., Detlefs, B., Wilkins, S. B., Beale, T. A. W., Mazzoli, C., Joly, Y., Hatton, P. D., Lorenzo, J. E. \& Brabers, V. A. M., (2009). J. Phys.: Condens. Matter 21, doi:10.1088/09538984/21/48/485601.

Blasco, J., García, J., Subías, G., Renevier, H., Stingaciau, M., Conder, K. \& Herrero-Martín, J., (2008a). Phys. Rev. B 78, doi:10.1103/PhysRevB.78.054123.

Blasco, J., Sánchez, M. C., García, J., Stankiewicz, J. \& Herrero-Martín, J., (2008b). J. Cryst. Growth 310, 3247-3250.

Blasco, J., García, J. \& Subías, G., (2011). Phys. Rev. B 83, doi:10.1103/PhysRevB.83.104105.

Brese, E. \& O’Keeffe, M., (1991). Acta Cryst. B47, 192-197. 
De Teresa, J. M., Fernández-Pacheco, A., Morellón, L., Orna, J., Pardo, J. A., Serrate, D., Algarabel, P. A. \& Ibarra, M. R., (2007). Microelectron. Eng. 84, 1660-1664.

Dmitrienko, V. E., Ishida, K., Kirfel, A. \& Ovchinnikova, E. N., (2005). Acta Cryst. A61, 481-493. García, J. \& Subías, G., (2004). J. Phys.: Condens. Matter 16, R145-R178. See the review of the experimental research on magnetite over the last 60 years and references therein.

García, J., Subías, G., Blasco, J., Proietti, M. G. \& Renevier, H., (2007). Comment on resonant X-ray diffraction studies on the charge ordering in magnetite, arXiv:cond-mat/0702135v2.

García, J., Subías, G., Herrero-Martín, J., Blasco, J., Cuartero, V., Sánchez, M. C., Mazzoli, C. \& Yakhou, F., (2009). Phys. Rev. Lett. 102, doi:10.1103/PhysRevLett.102.176405.

García, J., Subías, G., Proietti, M. G., Blasco, J., Renevier, H., Hodeau, J. L. \& Joly, Y., (2001). Phys. Rev. B 63, doi:10.1103/PhysRevB.63.054110.

García, J., Subías, G., Proietti, M. G., Renevier, H., Joly, Y., Hodeau, J. L., Blasco, J., Sánchez, M. C. \& Berar, J. F., (2000). Phys. Rev. Lett. 85, 578-581.

Goff, R. J., Wright, J. P., Attfield, J. P. \& Radaelli, P. G., (2005). J. Phys. Condens. Matter 17, 76337642 .

Huang, D. J., Lin, H. J., Okamoto, J., Chao, K. S., Jeng, H. T., Guo, G. Y., Hsu, C. H., Huang, C. M., Ling, D. C., Wu, W. B., Yang, C. S. \& Chen, C. T., (2006). Phys. Rev. Lett. 96, doi:10.1103/PhysRevLett.96.096401.

Iizumi, M., Koetzle, T. F., Shirane, G., Chikazumi, S., Matsui, M. \& Todo, S., (1982). Acta Cryst. B38, 2121-2133.

Imada, M., Fujimori, A. \& Tokura, Y., (1998). Rev. Mod. Phys. 70, 1039-1263.

Jeng, H. T., Guo, G. Y. \& Huang, D. J., (2004). Phys. Rev. Lett. 93,

doi:10.1103/PhysRevLett.93.156403.

Joly, Y., Lorenzo, J. E., Nazarenko, E., Hodeau, J. L., Mannix, D. \& Marin, C., (2008). Phys. Rev. B 78, doi:10.1103/PhysRevB.78.134110.

Kanazawa, M., Hagaiwara, K., Kokubun, J. \& Ishida, K., (2002). J. Phys. Soc. Japan 71, 1765-1770. Leonov, I., Yaresko, A. N., Antonov, V. N., Korotin, M. A. \& Anisimov, V. I., (2004). Phys. Rev. Lett. 93, doi:10.1103/PhysRevLett.93.146404.

Lorenzo, J. E., Mazzoli, C., Jaouen, N., Detlefs, C., Mannix, D., Grenier, S., Joly, Y. \& Marin, C., (2008). Phys. Rev. Lett. 101, doi:10.1103/PhysRevLett.101.226401.

Madsen, G. K. H. \& Novák, P., (2005). Europhys. Lett. 69, 777-783.

Nazarenko, E., Lorenzo, J. E., Joly, Y., Hodeau, J. L., Mannix, D. \& Marin, C., (2006). Phys. Rev. Lett. 97, doi:10.1103/PhysRevLett.97.056403.

Novák, P., Stepankova, H., Englich, J., Kohout, J. \& Brabers, V. A. M., (2000) Phys. Rev. B 61, 12561260.

Orna, J., Algarabel, P. A., Morellón, L., Pardo, J. A., de Teresa, J. M., López Antón, R., Bartolomé, F., García, L. M., Bartolomé, J., Cezar, J. C. \& Wildes, A., (2010). Phys. Rev. B 81, doi:10.1103/PhysRevB.81.144420. 
Paolasini, L., Detlefs, C., Mazzoli, C., Wilkins, S., Deen, P. P., Bombardi, A., Kernavanois, N., de Bergevin, F., Yakhou, F., Valade, J. P., Breslavetz, I., Fondacaro, A., Pepellin, G. \& Bernard, P., (2007). J. Synchrotron Rad. 14, 301-312.

Piekarz, P., Parlinski, K. \& Olés, A. M., (2007). Phys. Rev. B 76, doi:10.1103/PhysRevB.76.165124. Pinto, H. P. \& Elliot, S. D., (2006). J. Phys.: Condens. Matter 18, 10427-10436.

Proietti, M. G., Renevier, H., Hodeau, J. L., García, J., Bérar, J. F. \& Wolfers, P., (1999). Phys. Rev. B 59, 5479-5492.

Rowan, A. D., Patterson, C. H. \& Gasparov, L. V., (2009). Phys. Rev. B 79, doi:10.1103/Phys.RevB.79.205103.

Schlappa, J., Schüssler-Langeheine, C., Chang, C. F., Ott, H., Tanaka, A., Hu, Z., Haverkort, M. W., Schierle, E., Weschke, E., Kaindl, G. \& Tjeng, L. H., (2008). Phys. Rev. Lett. 100, doi:10.1103/PhysRevLett.100.026406.

Shepherd, J. P., Koetnizer, J. W., Aragón, R., Spalek, J. \& Honig, J. M., (1991). Phys. Rev. B 43, 84618471.

Subías, G., García, J. \& Blasco, J., (2005). Phys. Rev. B 71, doi:10.1103/PhysRevB.71.155103.

Subías, G., García, J., Blasco, J., Herrero-Martín, J. \& Sánchez, M. C., (2009). J. Phys.:Conference Series 190, doi:10.1088/1742-6596/190/1/012085.

Subías, G., García, J., Blasco, J., Proietti, M. G., Renevier, H. \& Sánchez, M. C., (2004a). Phys. Rev. Lett. 93, doi:10.1103/PhysRevLett.93.156408.

Subías, G., García, J., Proietti, M. G., Blasco, J., Renevier, H., Hodeau, J. L. \& Sánchez, M. C., (2004b). Phys. Rev. B 70, doi:10.1103/Phys.RevB.70.155105.

Szotek, Z., Temmerman, W. M., Svane, A., Petit, L., Stocks, G. M. \& Winter, H., (2003). Phys. Rev. B 68, doi:10.1103/Phys.RevB.68.054415.

Verwey, E. J. W., (1939). Nature (London) 144, 327-328.

Verwey, E. J. W. \& Haayman, P. W., (1941). Physica (Amsterdam) 9, 979-987.

Wilkins, S. B., Di Matteo, S., Beale, T. A. W., Joly, Y., Mazzoli, C., Hatton, P. D., Bencok, P., Yakhou, F. \& Brabers, V. A. M., (2009). Phys. Rev. B 79, doi:10.1103/Phys.RevB.79.201102.

Wright, J. P., Attfield, J. P. \& Radaelli, P. G., (2001). Phys. Rev. Lett. 87, doi:10.1103/PhysRevLett.87.266401.

Wright, J. P., Attfield, J. P. \& Radaelli, P. G., (2002). Phys. Rev. B 66, doi:10.1103/Phys.RevB.33.214422. 
Figure 1

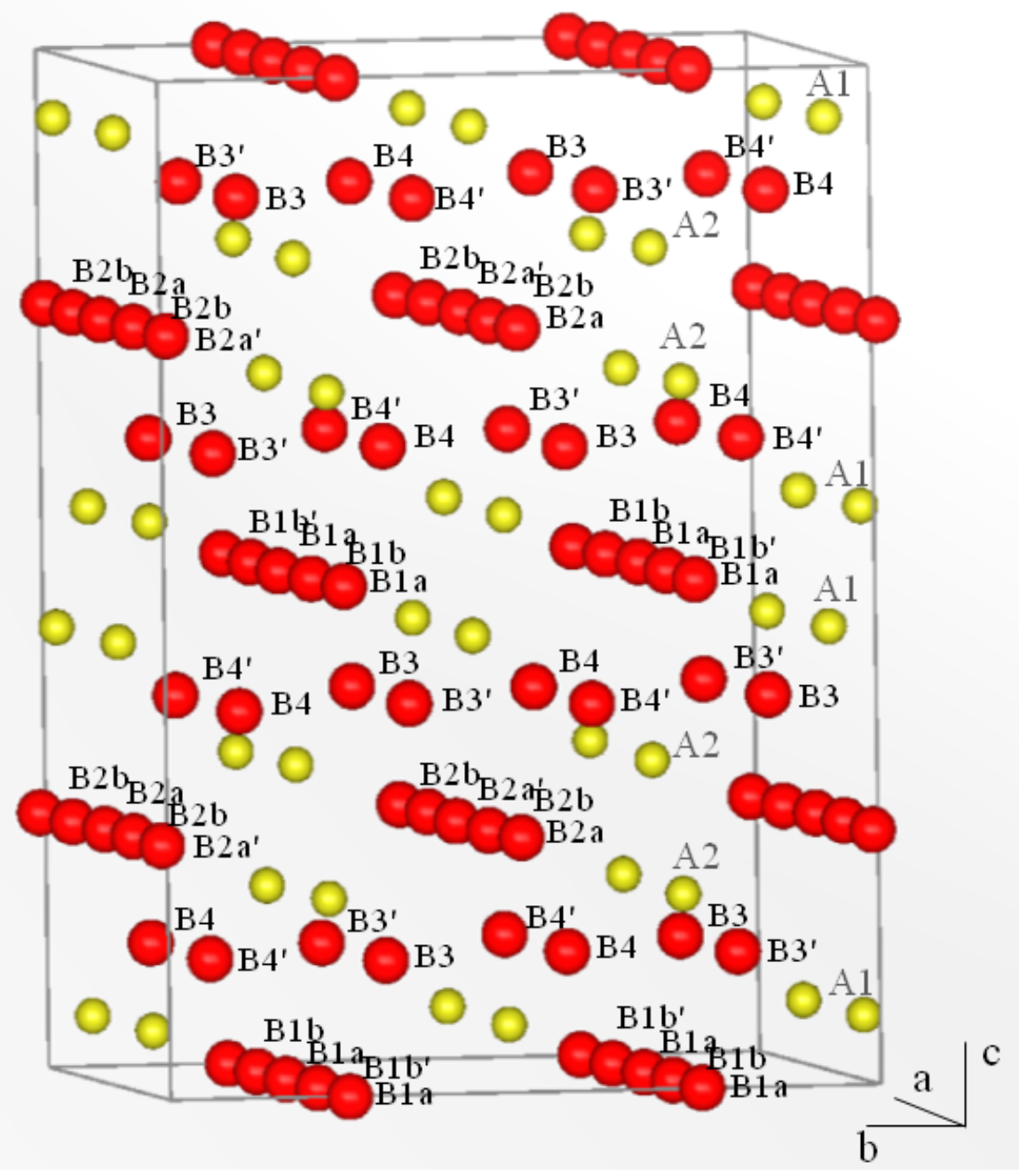


Figure 2

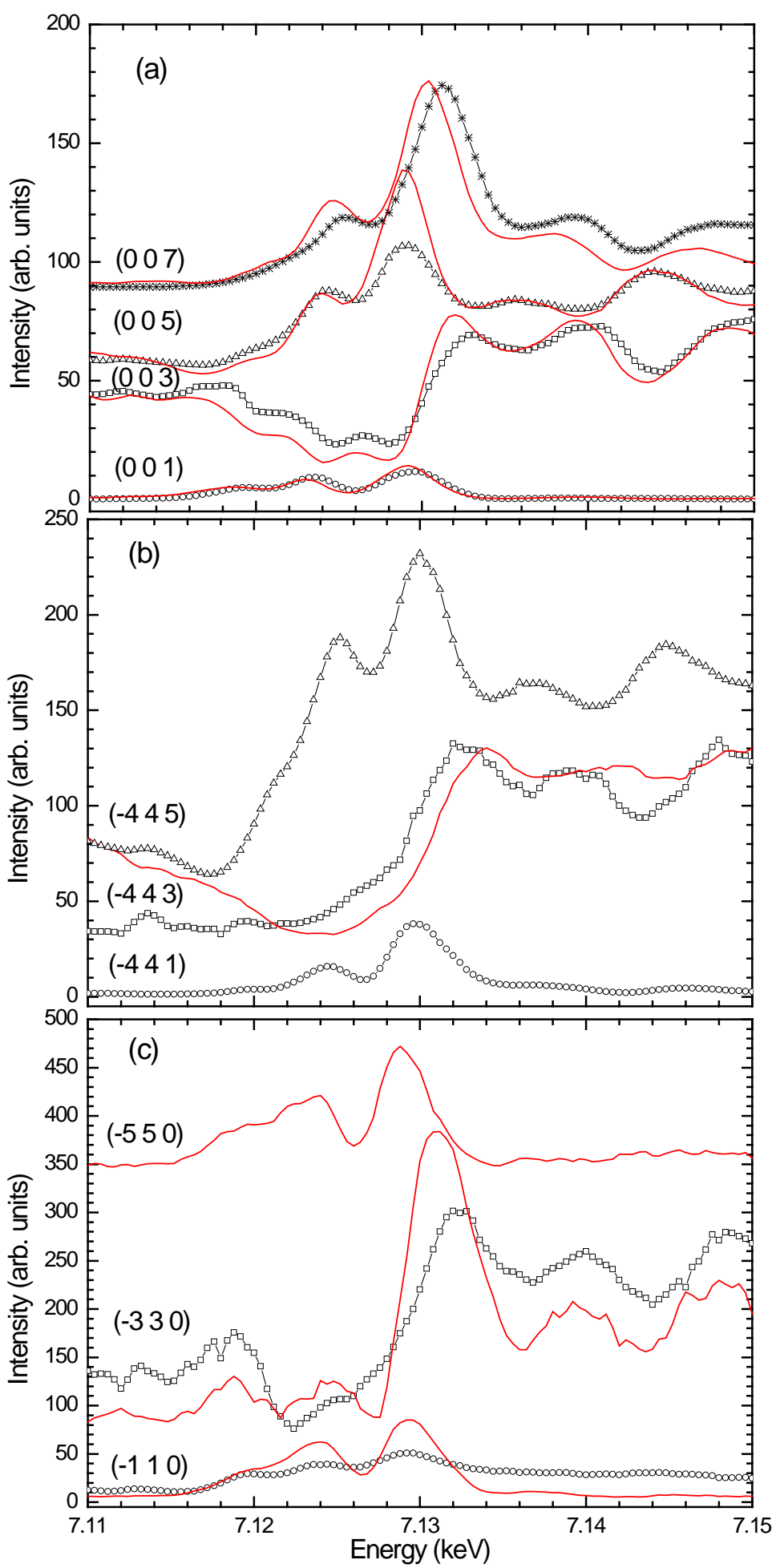


Figure 3
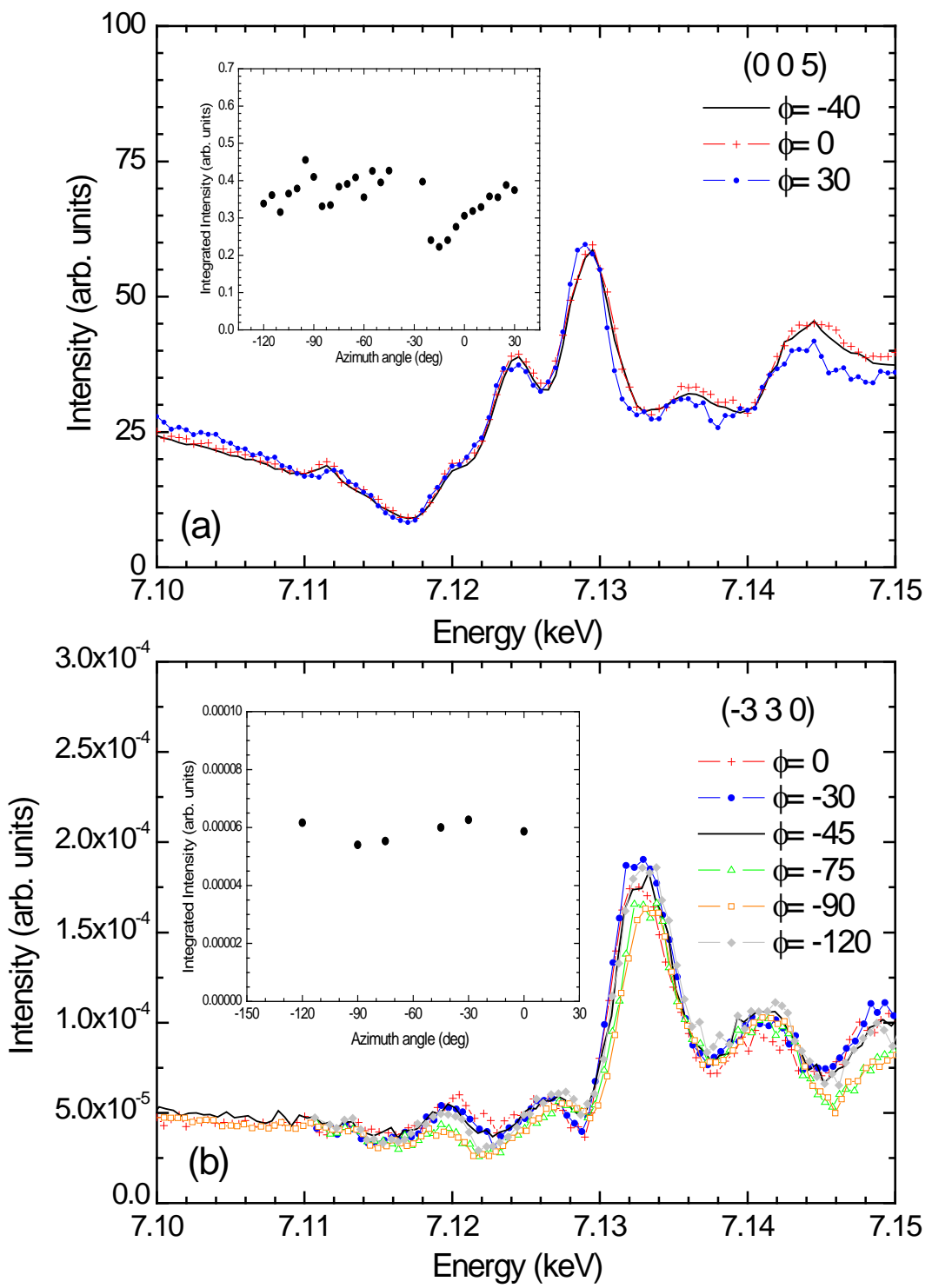
Figure 4

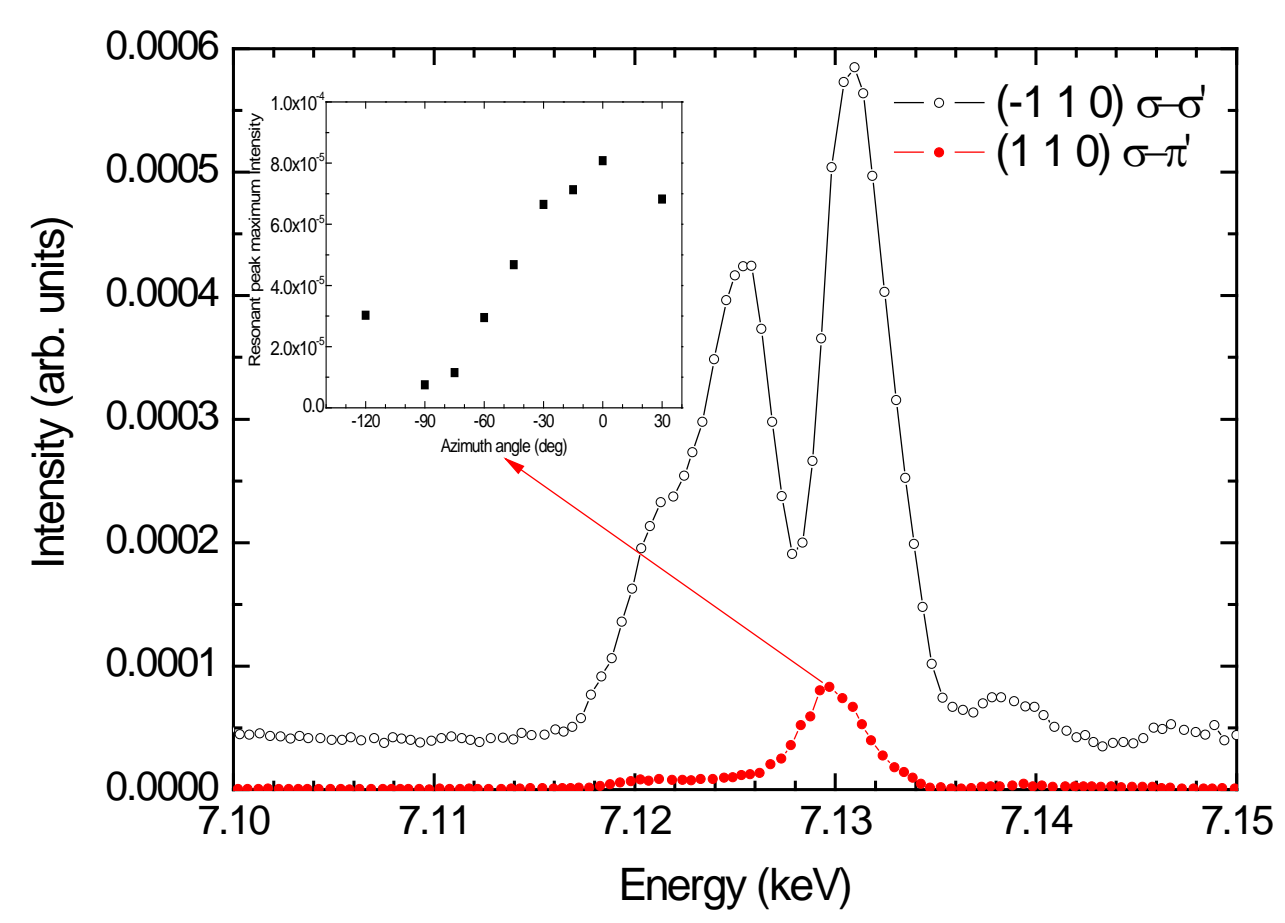


Figure 5
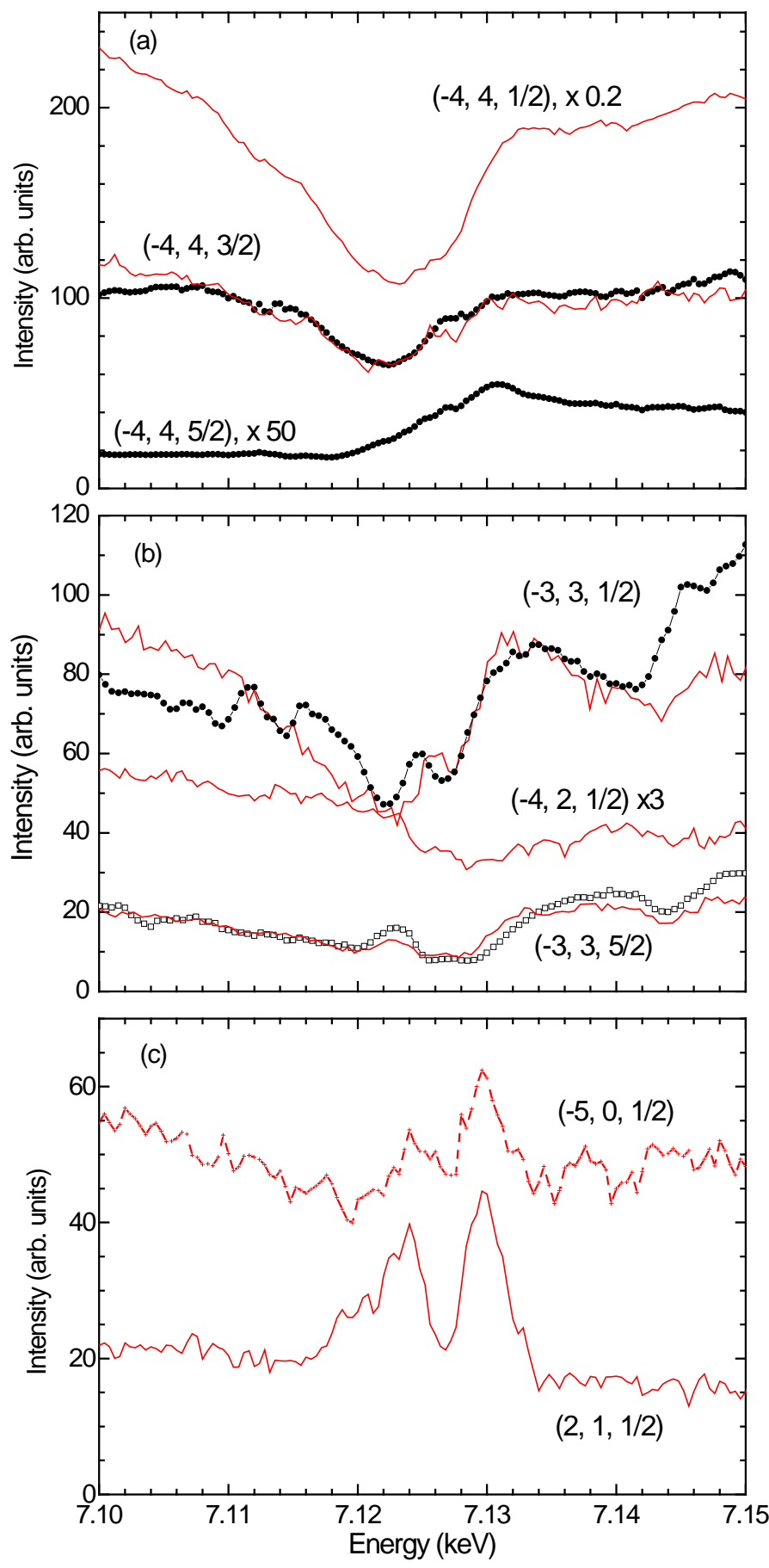
Figure 6
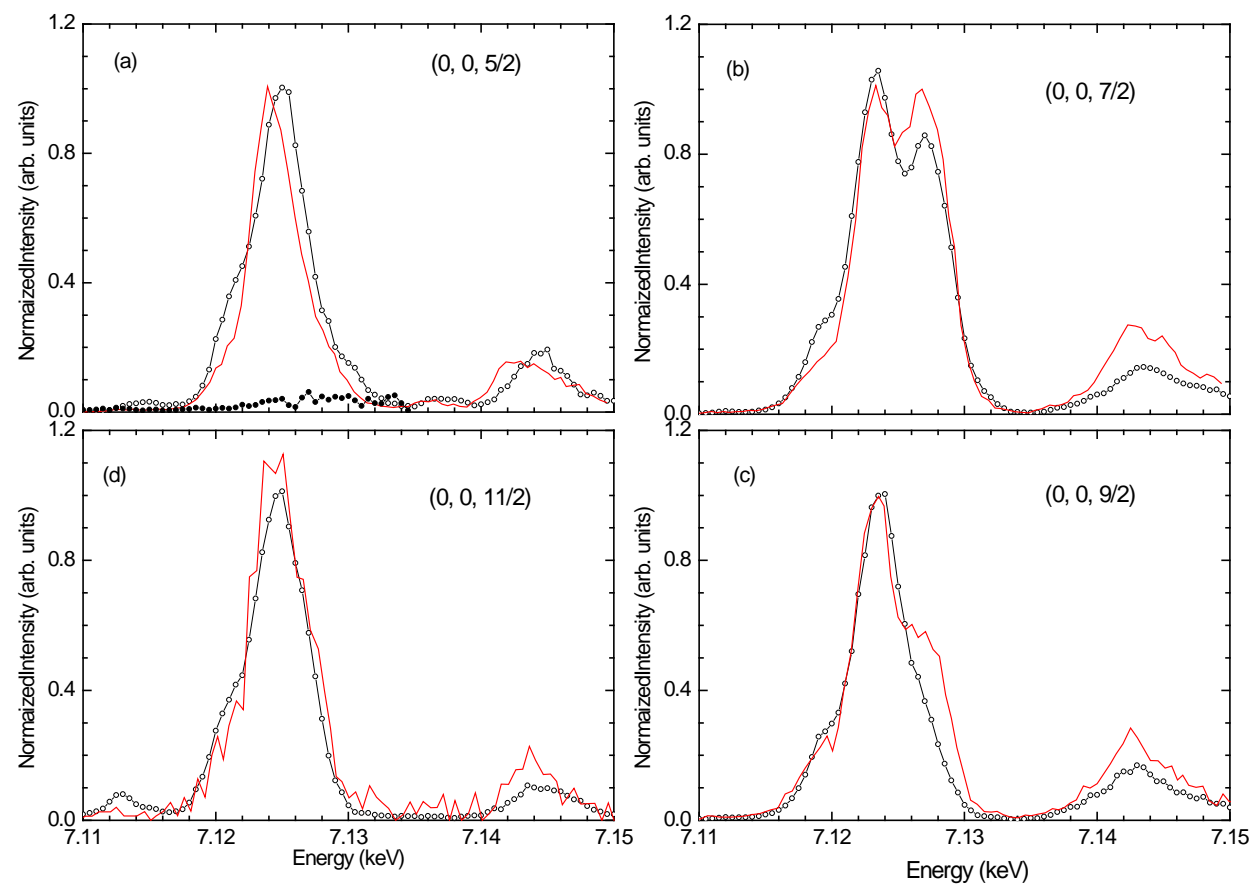
Figure 7

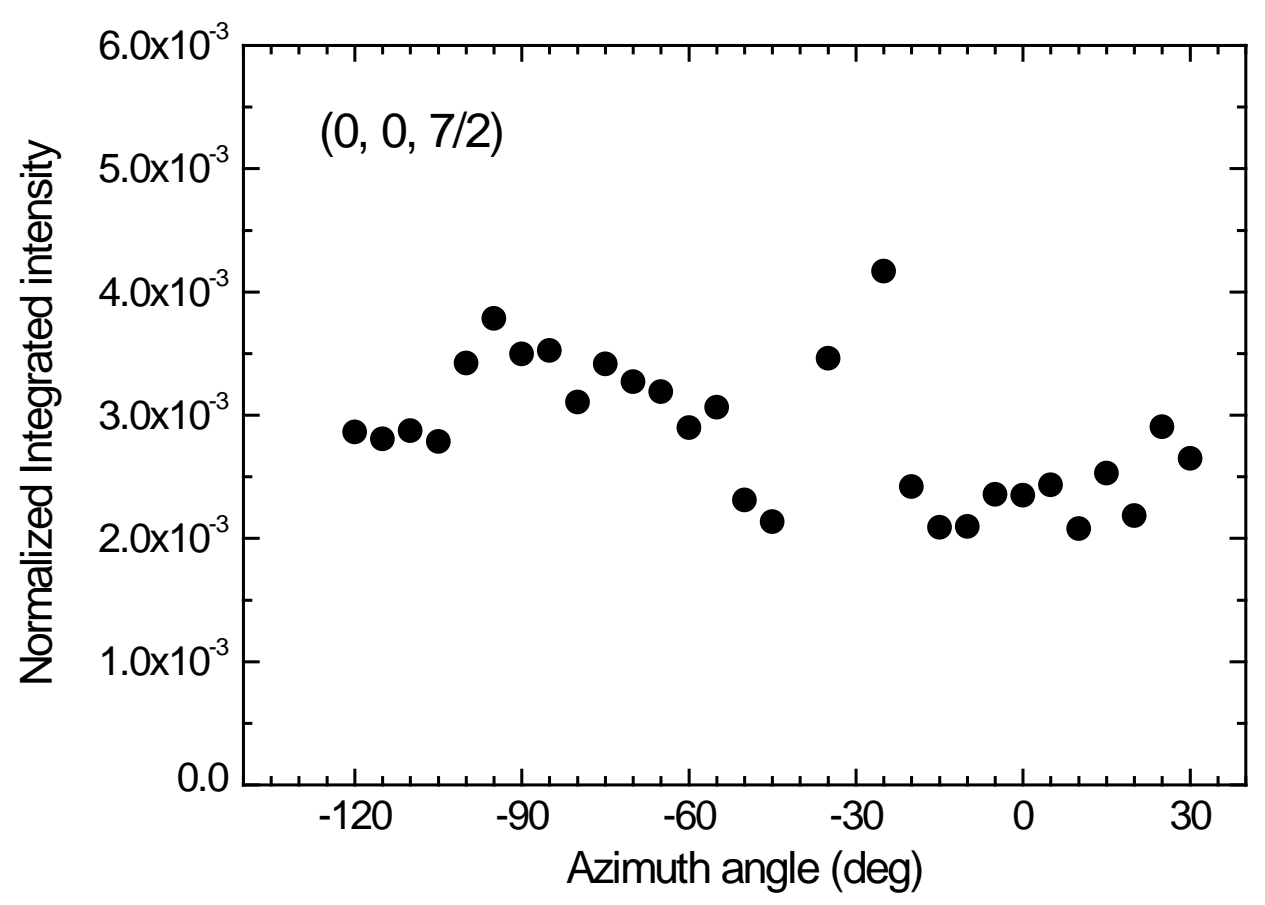


Figure 8

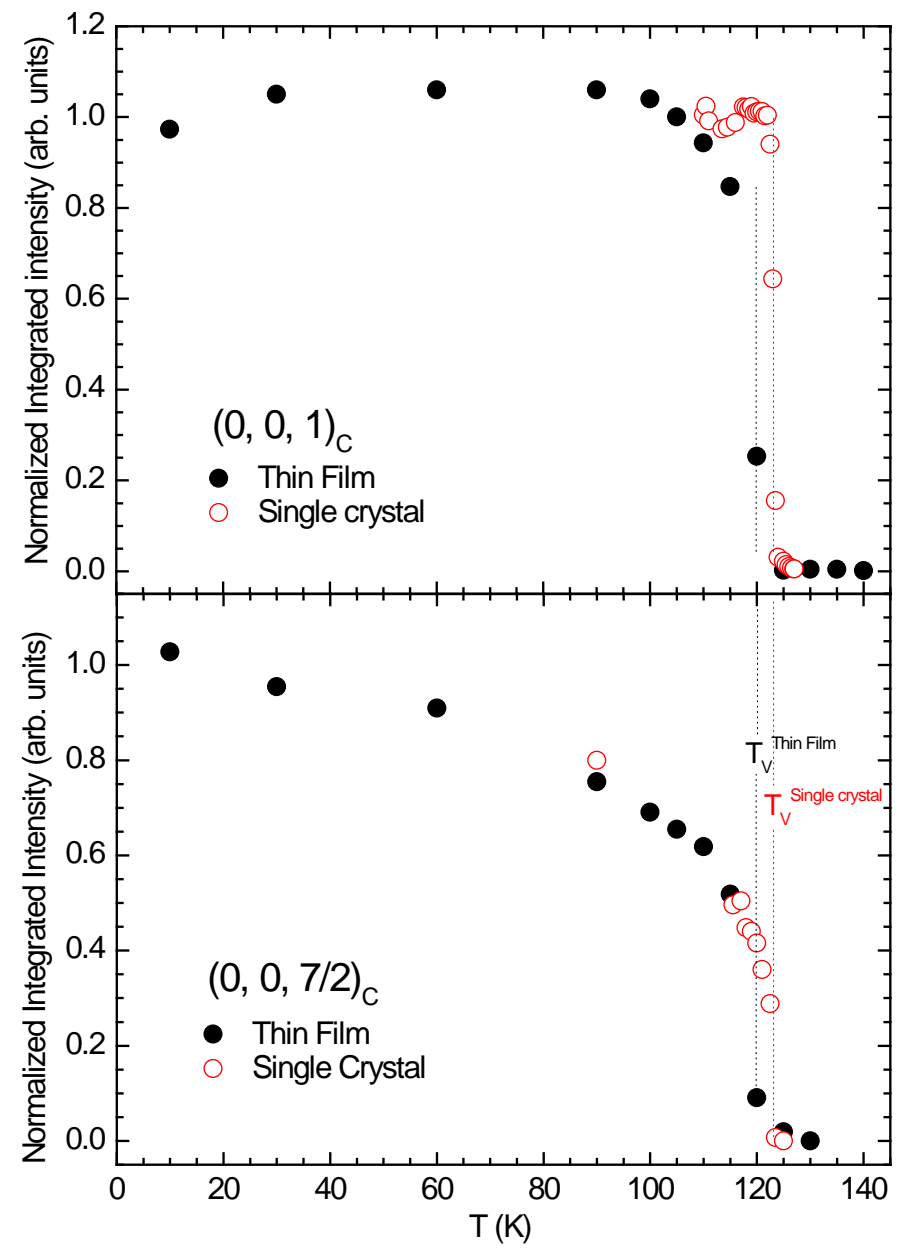


Figure 9
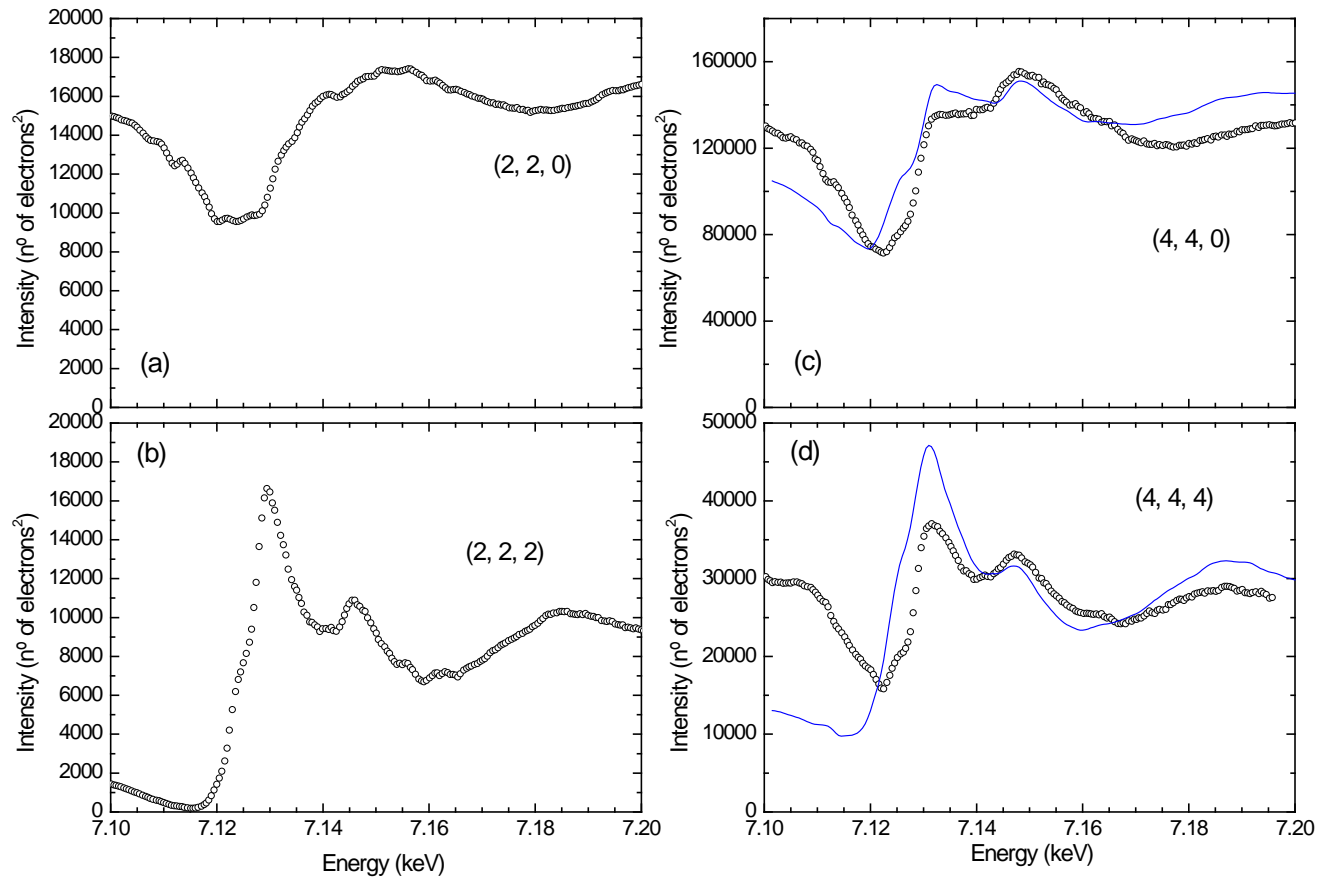
Figure 10

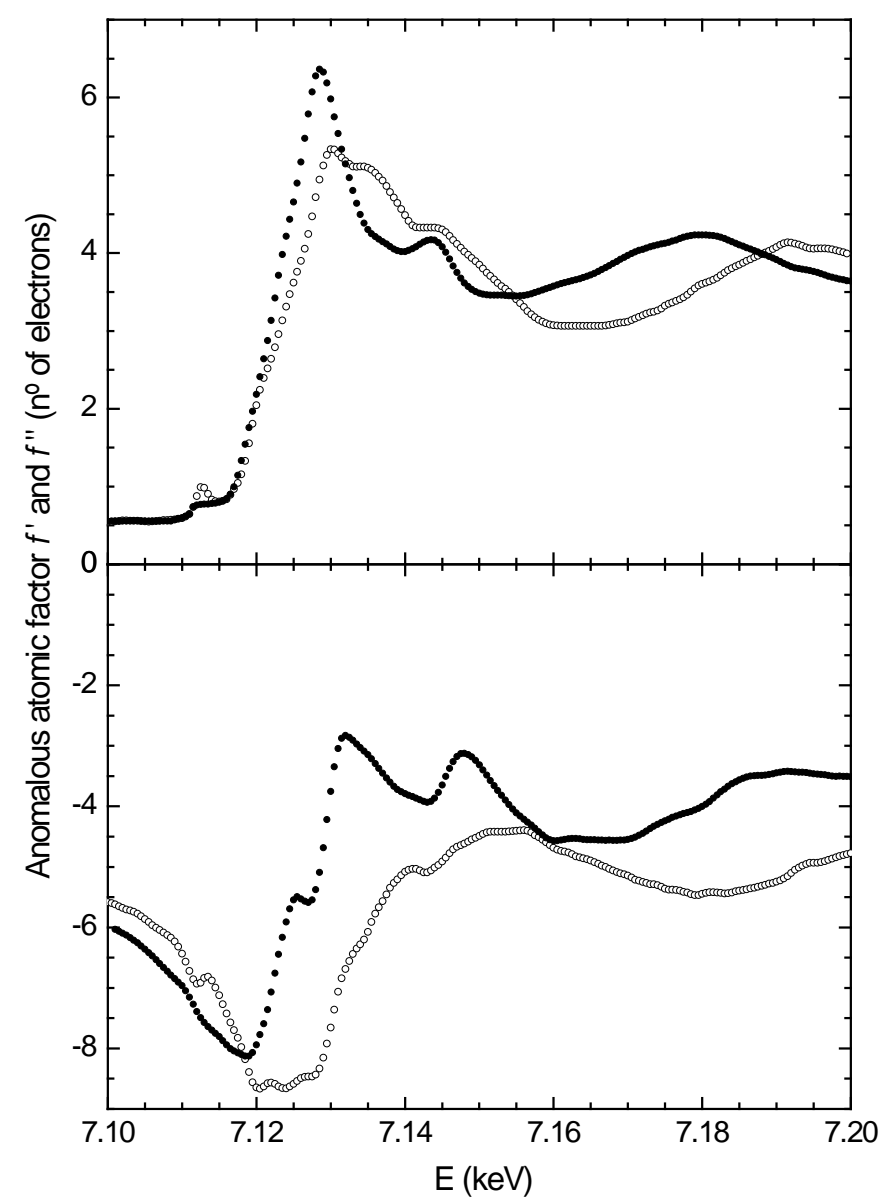


Figure 11

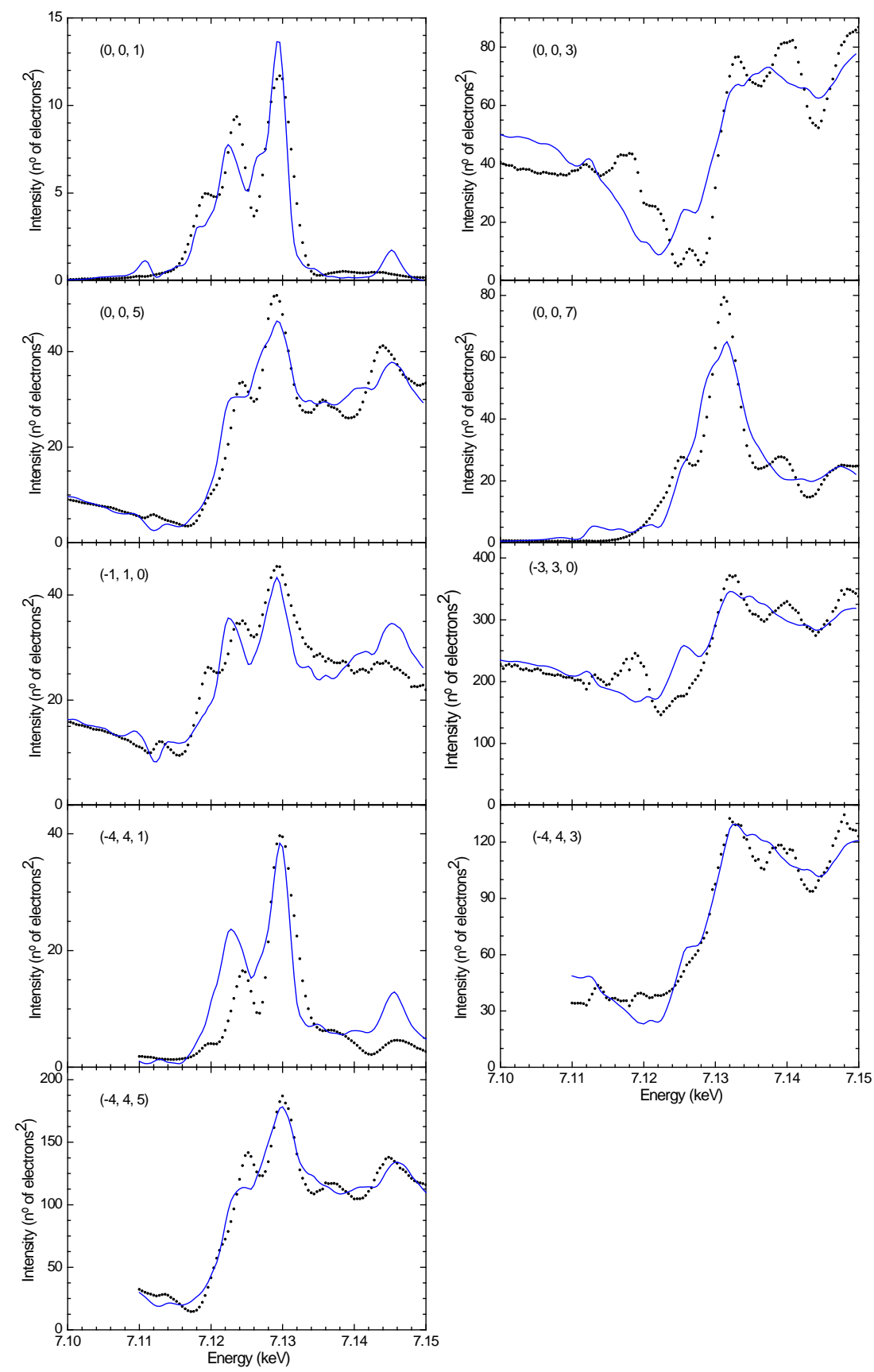


Figure 12

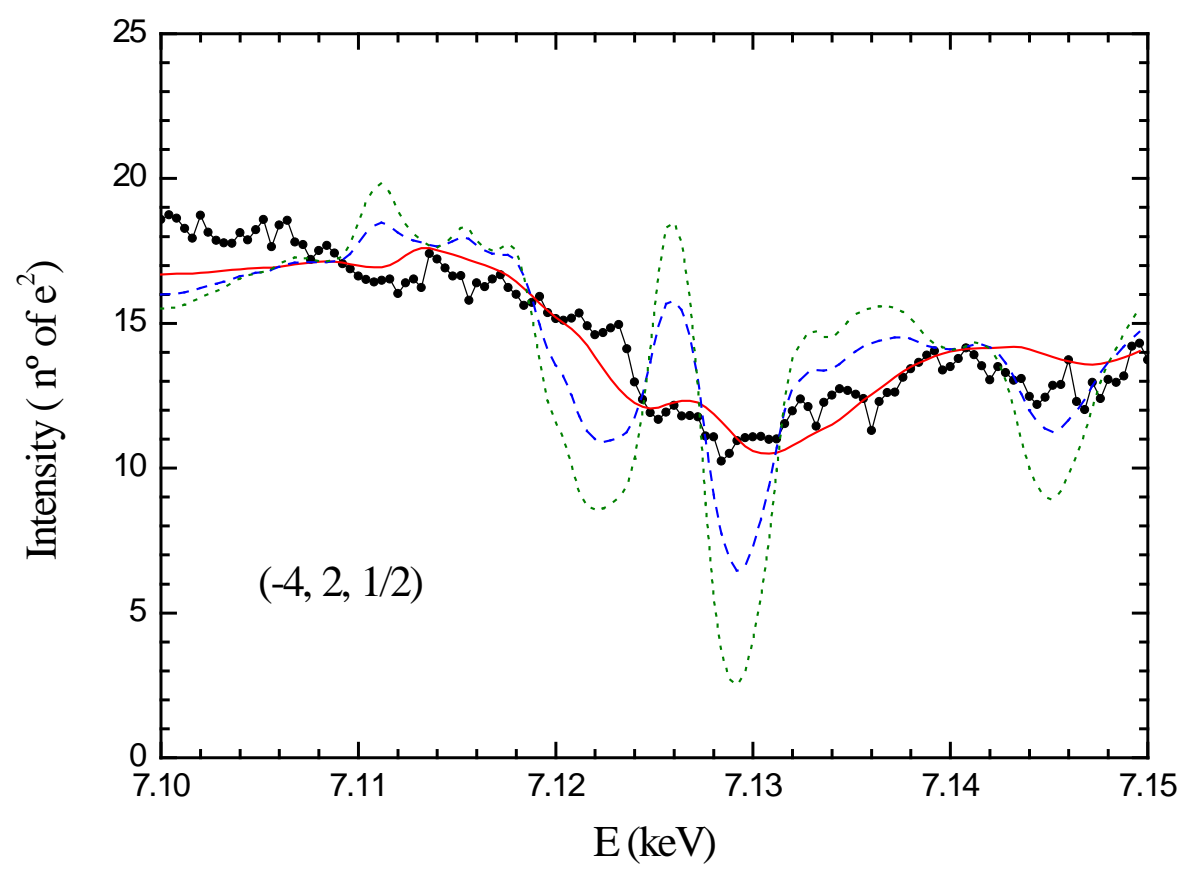


Figure 13
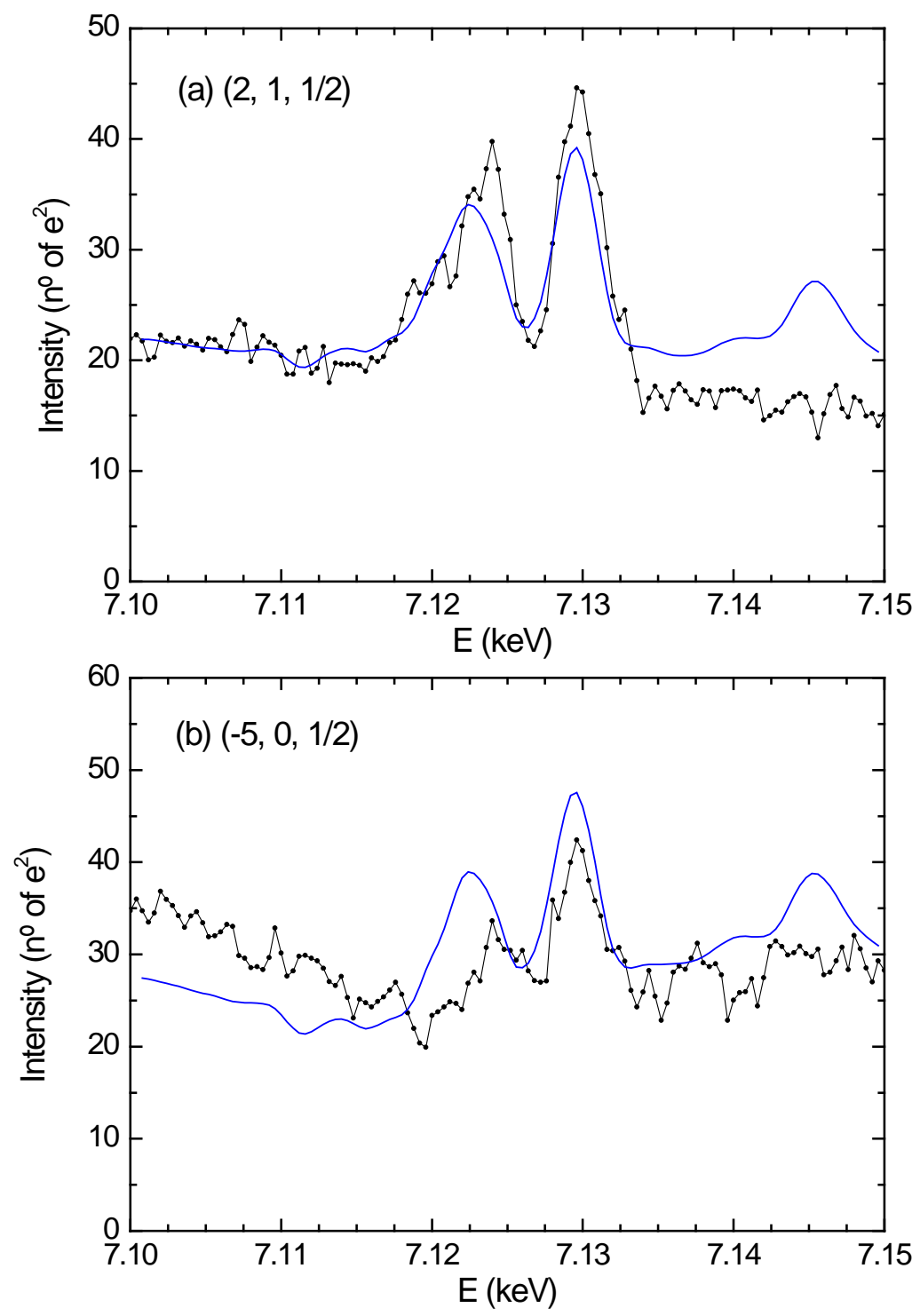